Revista de Matemática: Teoría y ApliCACiones 2018 25(1) : 1-28

CIMPA - UCR ISSN: 1409-2433 (PRINT), 2215-3373 (ONLINE)

\title{
ON DELTA-GRAPHS AND DELTA CONJECTURE
}

\author{
SOBRE DELTA-GRAFOS Y LA CONJETURA DELTA
}

\author{
PEDRo DíAZ NAVARRO*
}

Received: 18/Oct/2016; Revised: 09/Oct/2017;

Accepted: $17 /$ Oct/2017

\begin{abstract}
Revista de Matemática: Teoría y Aplicaciones is licensed under a Creative Commons Reconocimiento-NoComercial-Compartirigual 4.0 International License.

Creado a partir de la obra en http://www.revistas.ucr.ac.cr/index.php/matematica
\end{abstract}

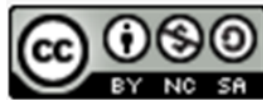

*Escuela de Matemática, Universidad de Costa Rica, San José, Costa Rica. E-Mail: pdiaz@costarricense.cr; pdiazn@gmail.com 


\begin{abstract}
In this paper we define two infinite families of graphs called $\mathrm{C}-\delta$ graphs and $\delta$-graphs and prove that $\delta$-graphs satisfy delta conjecture. Also we see that $\mathrm{C}-\delta$ graphs family contains the complements of $\delta$-graphs. Finally we give a list of $\mathrm{C}-\delta$ graphs and the relationship with the minimum semidefinite rank of these graphs.
\end{abstract}

Keywords: delta conjecture; simple connected graphs; minimum semidefinite rank; $\delta$-graph; C- $\delta$ graphs; orthogonal representation.

\title{
Resumen
}

En este artículo definimos dos familias de grafos llamadas $\mathrm{C}-\delta$ grafos y $\delta$-grafos y se prueba que los $\delta$-grafos satisfacen la conjetura delta. También vemos que la familia de los $\mathrm{C}-\delta$ grafos contienen los complementos de $\operatorname{los} \delta$-grafos. Finalmente damos una lista de $\mathrm{C}-\delta$ grafos y la relación con el rango mínimo semidefinido de estos grafos.

Palabras clave: conjetura delta; grafo simple conexo; rango mínimo semidefinido; $\delta$-grafo; C- $\delta$ grafo; representación ortogonal.

Mathematics Subject Classification: 05C50, 05C76, 05C85, 68R05, 65F99, 97K30.

\section{Introduction and preliminaries}

A graph $G$ consists of a set of vertices $V(G)=\{1,2, \ldots, n\}$ and a set of edges $E(G)$, where an edge is defined to be an unordered pair of vertices. The order of $G$, denoted $|G|$, is the cardinality of $V(G)$. A graph is simple if it has no multiple edges or loops. The complement of a graph $G(V, E)$ is the graph $\bar{G}=(V, \bar{E})$, where $\bar{E}$ consists of all those edges of the complete graph $K_{|G|}$ that are not in $E$. We say that two vertices of a graph $G$ are adjacent, denoted $v_{i} \sim v_{j}$, if there is an edge $\left\{v_{i}, v_{j}\right\}$ in $G$. Otherwise we say that the two vertices $v_{i}$ and $v_{j}$ are non-adjacent and we denote this by $v_{i} \nsim v_{j}$. Let $N(v)$ denote the set of vertices that are adjacent to the vertex $v$ and let $N[v]=\{v\} \cup N(v)$. The degree of a vertex $v$ in $G$, denoted $\mathrm{d}_{G}(v)$, is the cardinality of $N(v)$. We use $\delta(G)$ to denote the minimum degree of the vertices in $G$, whereas $\Delta(G)$ will denote the maximum degree of the vertices in $G$. If $\mathrm{d}_{G}(v)=1$, then $v$ is said to be a pendant vertex of $G$.

A subgraph $H=(V(H), E(H))$ of $G=(V, E)$ is a graph with $V(H) \subseteq$ $V(G)$ and $E(H) \subseteq E(G)$. An induced subgraph $H$ of $G$, denoted $\mathrm{G}[\mathrm{V}(\mathrm{H})]$, is a subgraph with $V(H) \subseteq V(G)$ and $E(H)=\{\{i, j\} \in E(G): i, j \in V(H)\}$. Sometimes we denote the edge $\{i, j\}$ as $i j$. 
Two graphs $G(V, E)$ and $H\left(V^{\prime}, E^{\prime}\right)$ are identical denoted $G=H$, if $V=V^{\prime}, E=E^{\prime}$.

A complete graph is a simple graph in which the vertices are pairwise adjacent. Denote by $K_{n}$ the complete graph on $n$ vertices. Complete graph $K_{n}$ are also called a clique. The clique cover number of a graph $G$, denoted by $\operatorname{cc}(G)$ is the minimum number of cliques in $G$ needed to cover the vertex set of $\mathrm{G}$. We will use $n G$ to denote $n$ copies of a graph $G$. For example, $3 K_{1}$ denotes three isolated vertices $K_{1}$ while $2 K_{2}$ is the graph given by two disconnected copies of $K_{2}$.

A path is a list of distinct vertices in which successive vertices are connected by edges. A path on $n$ vertices is denoted by $P_{n}$. A graph $G$ is said to be connected if there is a path between any two vertices of $G$. A cycle on $n$ vertices, denoted by $C_{n}$, is a path such that the beginning vertex and the end vertex are the same. A tree is a connected graph with no cycles. A graph $G(V, E)$ is said to be chordal if it has no induced cycles $C_{n}$ with $n \geq 4$. A component of a graph $G(V, E)$ is a maximal connected subgraph. A cut vertex is a vertex whose deletion increases the number of components.

The union $G_{1} \cup G_{2}$ of two graphs $G_{1}\left(V_{1}, E_{1}\right)$ and $G_{2}\left(V_{2}, G_{2}\right)$ is the union of their vertex set and edge set, that is $G_{1} \cup G_{2}\left(V_{1} \cup V_{2}, E_{1} \cup E_{2}\right)$. When $V_{1}$ and $V_{2}$ are disjoint their union is called disjoint union and denoted $G_{1} \sqcup G_{2}$.

Further details can be found in $[8,9,14]$.

A square matrix $A=\left[a_{i j}\right]$ is combinatorially symmetric when $a_{i j}=0$ if and only if $a_{j i}=0$. We say that $G(A)$ is the graph of a combinatorially symmetric matrix $A=\left[a_{i j}\right]$ if $V=\{1,2, \ldots, n\}$ and $E=\left\{\{i, j\}: a_{i j} \neq\right.$ $0\}$. The main diagonal entries of $A$ play no role in determining $G$. Define $S(G, \mathbb{F})$ as the set of all $n \times n$ matrices that are real symmetric if $\mathbb{F}=\mathbb{R}$ or complex Hermitian if $\mathbb{F}=\mathbb{C}$ whose graph is $G$. The sets $S_{+}(G, \mathbb{F})$ are the corresponding subsets of positive semidefinite (psd) matrices. The smallest possible rank of any matrix $A \in S(G, \mathbb{F})$ is the minimum rank of $G$, denoted $\operatorname{mr}(G, \mathbb{F})$, and the smallest possible rank of any matrix $A \in S_{+}(G, \mathbb{F})$ is the minimum semidefinite rank of $G$, denoted $\mathrm{mr}_{+}(G)$ or $\operatorname{msr}(G)$.

In 1996, the minimum rank among real symmetric matrices with a given graph was studied by Nylen [27]. It gave rise to the area of minimum rank problems which led to the study of minimum rank among complex Hermitian matrices and positive semidefinite matrices associated with a given graph. Many results can be found, for example, in [1, 19, 23, 24, 27]. 
During the American Institute of Mathematics workshop of 2006 in Palo Alto, CA, it was stablished the following conjecture:

Conjecture 1.1. For any graph $G$ and infinite field $\mathbb{F}, \operatorname{mr}(G, \mathbb{F}) \leq|G|-\delta(G)$ where $\delta(G)$ is the minimum degree of $G$.

It was shown that if $\delta(G) \leq 3$ or $\delta(G) \geq|G|-2$ this inequality holds. Also it can be verified that if $|G| \leq 6$ then $\operatorname{mr}(G, F) \leq|G|-\delta(G)$. Also it was proven that any bipartite graph satisfies this conjecture. This conjecture is called the delta conjecture. If we restrict the study to consider matrices in $S_{+}(G, \mathbb{F})$, then delta conjecture is written as $\operatorname{msr}(G) \leq|G|-\delta(G)$. Clearly if the conjecture holds for $\operatorname{msr}(G)$ then it also holds for $\operatorname{mr}(G)$. Some results on delta conjecture can be found in $[7,13,26,30]$ but the general problem remains unsolved.

\section{The minimum semidefinite rank of a graph}

In this section we will establish some definitions and results for the minimum semidefinite rank (msr) of a graph $G$ that we will be using in the subsequent chapters.

A positive definite matrix $A$ is an Hermitian $n \times n$ matrix such that $x^{\star} A x>0$ for all nonzero $x \in \mathbb{C}^{n}$. Equivalently, $A$ is a $n \times n$ Hermitian positive definite matrix if and only if all the eigenvalues of $A$ are positive ([20], p. 250).

A $n \times n$ Hermitian matrix $A$ such that $x^{\star} A x \geq 0$ for all $x \in \mathbb{C}^{n}$ is said to be positive semidefinite (psd). Equivalently, $A$ is a $n \times n$ Hermitian positive semidefinite matrix if and only if $A$ has all eigenvalues nonnegative ([20], p. 182).

If $\vec{V}=\left\{\overrightarrow{v_{1}}, \overrightarrow{v_{2}}, \ldots, \overrightarrow{v_{n}}\right\} \subset \mathbb{R}^{m}$ is a set of column vectors then the matrix $A^{T} A$, where $A=\left[\begin{array}{llll}\overrightarrow{v_{1}} & \overrightarrow{v_{2}} & \ldots & \overrightarrow{v_{n}}\end{array}\right]$ and $A^{T}$ represents the transpose matrix of $A$, is a psd matrix called the Gram matrix of $\vec{V}$. Let $G(V, E)$ be a graph associated with this Gram matrix. Then $V_{G}=\left\{v_{1}, \ldots, v_{n}\right\}$ correspond to the set of vectors in $\vec{V}$ and $\mathrm{E}(\mathrm{G})$ correspond to the nonzero inner products among the vectors in $\vec{V}$. In this case $\vec{V}$ is called an orthogonal representation of $G(V, E)$ in $\mathbb{R}^{m}$. If such an orthogonal representation exists for $G$ then $\operatorname{msr}(G) \leq m$. 
Some of the most common results about the minimum semidefinite rank of a graph are the following:

Result 2.1 ([19]). If $T$ is a tree then $\operatorname{msr}(T)=|T|-1$.

Result 2.2 ([11]). The cycle $C_{n}$ has minimum semidefinite rank $n-2$.

Result 2.3 ([11]). If a connected graph $G$ has a pendant vertex $v$, then $\operatorname{msr}(G)=$ $\operatorname{msr}(G-v)+1$ where $G-v$ is obtained as an induced subgraph of $G$ by deleting $v$.

Result 2.4 ([18]). If $G$ is a connected, chordal graph, then $\operatorname{msr}(G)=\operatorname{cc}(G)$.

Result 2.5 ([10]). If a graph $G(V, E)$ has a cut vertex, so that $G=G_{1} \cdot G_{2}$, then $\operatorname{msr}(G)=\operatorname{msr}\left(G_{1}\right)+\operatorname{msr}\left(G_{2}\right)$.

\section{$3 \delta$-graphs and the delta conjecture}

In this section we define a new family of graphs called $\delta$-graphs and show that they satisfy the delta conjecture.

Definition 3.1. Suppose that $G=(V, E)$ with $|G|=n \geq 4$ is simple and connected such that $\bar{G}=(V, \bar{E})$ is also simple and connected. We say that $G$ is a $\delta$-graph if we can label the vertices of $G$ in such a way that

(1) the induced graph of the vertices $v_{1}, v_{2}, v_{3}$ in $G$ is either $3 K_{1}$ or $K_{2} \sqcup K_{1}$, and

(2) for $m \geq 4$, the vertex $v_{m}$ is adjacent to all the prior vertices $v_{1}, v_{2}, \ldots, v_{m-1}$ except for at most $\left\lfloor\frac{m}{2}-1\right\rfloor$ vertices.

Definition 3.2. Suppose that a graph $G(V, E)$ with $|G|=n \geq 4$ is simple and connected such that $\bar{G}=(V, \bar{E})$ is also simple and connected. We say that $G(V, E)$ is a $\mathbf{C}$ - $\delta$ graph if $\bar{G}$ is a $\delta$-graph.

In other words, $G$ is a $\mathbf{C}$ - $\delta$ graph if we can label the vertices of $G$ in such a way that

(1) the induced graph of the vertices $v_{1}, v_{2}, v_{3}$ in $G$ is either $K_{3}$ or $P_{3}$, and

(2) for $m \geq 4$, the vertex $v_{m}$ is adjacent to at most $\left\lfloor\frac{m}{2}-1\right\rfloor$ of the prior vertices $v_{1}, v_{2}, \ldots, v_{m-1}$. 

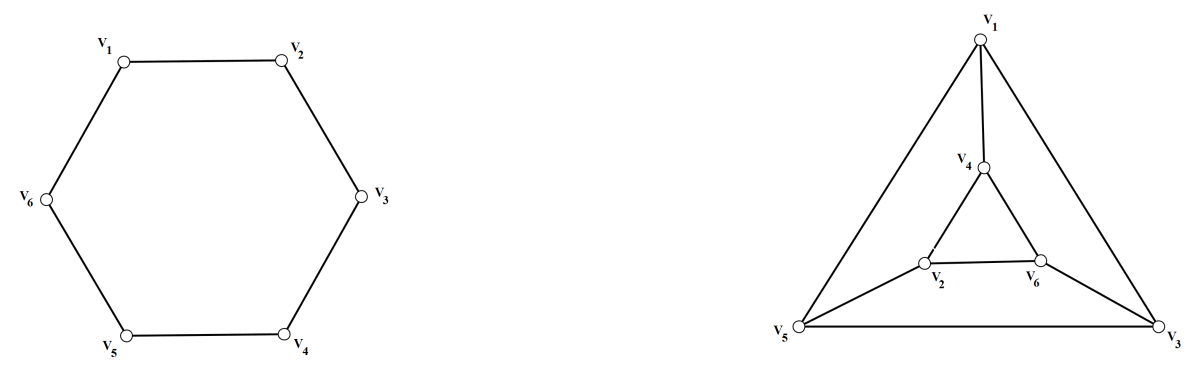

Figure 1: The graphs $C_{6}$ and the 3-prism.

Example 3.3. The cycle $C_{n}, n \geq 6$ is a $\mathrm{C}-\delta$ graph and its complement is a $\delta$-graph.

Note that we can label the vertices of $C_{6}$ clockwise $v_{1}, v_{2}, v_{3}, v_{4}, v_{5}, v_{6}$ (see Figure 1). The graph induced by $v_{1}, v_{2}, v_{3}$ is $P_{3}$. The vertex $v_{4}$ is adjacent to a prior vertex which is $v_{3}$. Also, the vertex $v_{5}$ is adjacent to vertex $v_{4}$ and the vertex $v_{6}$ is adjacent to two prior vertices $v_{1}$ and $v_{5}$. Hence, $C_{6}$ is $\mathrm{C}-\delta$ graph. The 3-prism which is isomorphic to the complement of $C_{6}$, is a $\delta$-graph.

Lemma 3.4. Let $G(V, E)$ be a $\delta$-graph. Then the induced graph of $\left\{v_{1}, v_{2}, v_{3}\right\}$ in $G$ denoted by $H$ has an orthogonal representation in $\mathbb{R}^{\Delta(\bar{G})+1}$ satisfying the following conditions:

(i) the vectors in the orthogonal representation of $H$ can be chosen with nonzero coordinates, and

(ii) $\vec{v} \notin \operatorname{Span}(\vec{u})$ for each pair of distinct vertices $u, v$ in $H$.

Proof. Let $G(V, E)$ be a $\delta$-graph. Label the vertices of $G$ in such a way that the labeling satisfies the conditions (1) and (2) for $\delta$-graphs. Let $H$ be the induced subgraph in $G$ by $\left\{v_{1}, v_{2}, v_{3}\right\} \subseteq V$. Then $H$ is either $3 K_{1}$ or $K_{2} \sqcup K_{1}$. Since $G$ and $\bar{G}$ are simple and connected it follows that

$$
2 \leq \Delta(\bar{G}) \leq n-2
$$

Let $\left\{\vec{e}_{j}\right\}, j=1,2, \ldots, \Delta(\bar{G})+1$ be the standard orthonormal basis for $\mathbb{R}^{\Delta(\bar{G})+1}$. Case 1. Suppose the induced graph $H$ of $\left\{v_{1}, v_{2}, v_{3}\right\} \subseteq V$ in $G$ is $3 K_{1}$ which is disconnected. 
Choose $\overrightarrow{v_{1}}, \overrightarrow{v_{2}}, \overrightarrow{v_{3}}$ in $\mathbb{R}^{\Delta(\bar{G})+1}$ corresponding to $v_{1}, v_{2}, v_{3}$ respectively such that:

$$
\begin{aligned}
& \overrightarrow{v_{1}}=\sum_{j=1}^{\Delta(\bar{G})+1} k_{1, j} \vec{e}_{j} \\
& \overrightarrow{v_{2}}=\sum_{j=1}^{\Delta(\bar{G})+1} k_{2, j} \vec{e}_{j} \\
& \overrightarrow{v_{3}}=\sum_{j=1}^{\Delta(\bar{G})+1} k_{3, j} \vec{e}_{j}
\end{aligned}
$$

where the scalars $k_{1, j}, j=1,2, \ldots, \Delta(\bar{G})+1$, and $k_{2, s}, s=1,2, \ldots, \Delta(\bar{G})$ are chosen not zero from different field extensions in the following way:

$$
\begin{aligned}
& k_{1,1} \notin \mathbb{Q}, \\
& k_{1,2} \notin \mathbb{Q}\left[k_{1,1}\right], \\
& k_{1,3} \notin \mathbb{Q}\left[k_{1,1}, k_{1,2}\right], \\
& \vdots \\
& k_{1, \Delta(\bar{G})+1} \notin \mathbb{Q}\left[k_{1,1}, k_{1,2}, \ldots, k_{1, \Delta}(\bar{G})\right], \\
& k_{2,1} \notin \mathbb{Q}\left[k_{1,1}, k_{1,2}, \ldots, k_{1, \Delta(\bar{G})+1}\right], \\
& k_{2,2} \notin \mathbb{Q}\left[k_{1,1}, k_{1,2}, \ldots, k_{1, \Delta(\bar{G})+1}, k_{2,1}\right], \\
& \vdots \\
& k_{2, \Delta(\bar{G})} \notin \mathbb{Q}\left[k_{1,1}, k_{1,2}, \ldots, k_{1, \Delta(\bar{G})+1}, k_{2,1}, \ldots, k_{2, \Delta(\bar{G})-1}\right] .
\end{aligned}
$$

Now choose

$$
k_{2, \Delta(\bar{G})+1}=\frac{-1}{k_{1, \Delta(\bar{G})+1}} \sum_{j=1}^{\Delta(\bar{G})} k_{1, j} k_{2, j} .
$$

As a consequence $\left\langle\vec{v}_{1}, \vec{v}_{2}\right\rangle=0$. 
In order to find a vector $\vec{v}_{3}$, we need to solve the $2 \times(\Delta(\bar{G})+1)$ system satisfying

$$
\begin{aligned}
& \left\langle\vec{v}_{1}, \vec{v}_{3}\right\rangle=0 \\
& \left\langle\vec{v}_{2}, \vec{v}_{3}\right\rangle=0
\end{aligned}
$$

in the variables $k_{3, j}, j=1,2, \ldots, \Delta(\bar{G})+1$. The homogeneous system has infinitely many solutions because $\Delta(\bar{G})+1 \geq 3$. Reducing the matrix of this system to echelon form we get

$$
\begin{gathered}
\left(\begin{array}{ccccc}
k_{1,1} & k_{1,2} & k_{1,3} & \ldots & k_{1, \Delta}(\bar{G})+1 \\
k_{2,1} & k_{2,2} & k_{2,3} & \ldots & k_{2, \Delta(\bar{G})+1}
\end{array}\right) \sim \\
\left(\begin{array}{ccccc}
1 & \frac{k_{1,2}}{k_{1,1}} & \frac{k_{1,3}}{k_{1,1}} & \ldots & \frac{k_{1, \Delta(\bar{G})+1}}{k_{1,1}} \\
k_{2,1} & k_{2,2} & k_{2,3} & \ldots & k_{2, \Delta(\bar{G})+1}
\end{array}\right) \sim \\
\left(\begin{array}{ccccc}
1 & \frac{k_{1,2}}{k_{1,1}} & \frac{k_{1,3}}{k_{1,1}} & \ldots & \frac{k_{1, \Delta(\bar{G})+1}}{k_{1,1}} \\
0 & k_{2,2}-\frac{k_{2,1} k_{1,2}}{k_{1,1}} & k_{2,3}-\frac{k_{2,1} k_{1,3}}{k_{1,1}} & \ldots & k_{2, \Delta(\bar{G})+1}-\frac{k_{2,1} k_{1, \Delta}+1}{k_{1,1}}
\end{array}\right)
\end{gathered}
$$

Let $\alpha=k_{2,2}-\frac{k_{2,1} k_{1,2}}{k_{1,1}}$. Since $\alpha \neq 0$ because $k_{2,2} \notin \mathbb{Q}\left[k_{1,1}, \ldots, k_{1, \Delta}(\bar{G})+1, k_{2,1}\right]$. We can get the echelon form of the matrix by multiplying the second row by $\frac{1}{\alpha}$.

$$
\left(\begin{array}{ccccc}
1 & \frac{k_{1,2}}{k_{1,1}} & \frac{k_{1,3}}{k_{1,1}} & \ldots & \frac{k_{1, \Delta(\bar{G})+1}}{k_{1,1}} \\
0 & 1 & \frac{1}{\alpha}\left(k_{2,3}-\frac{k_{2,1} k_{1,3}}{k_{1,1}}\right) & \ldots & \frac{1}{\alpha}\left(k_{2, \Delta}(\bar{G})+1-\frac{k_{2, \Delta}(\bar{G})+1}{k_{1,1}}\right)
\end{array}\right)
$$

Since the system has infinitely many solutions, $k_{3, j}, j=3, \ldots, \Delta(\bar{G})+1$ are free parameters. We can choose them from different field extensions in the following way,

$k_{3,3} \notin \mathbb{Q}\left[k_{1,1}, k_{1,2}, \ldots, k_{1, \Delta(\bar{G})+1}, k_{2,1}, \ldots, k_{2, \Delta(\bar{G})+1}\right]$,

$k_{3,4} \notin \mathbb{Q}\left[k_{1,1}, k_{1,2}, \ldots, k_{1, \Delta(\bar{G})+1}, k_{2,1}, \ldots, k_{2, \Delta(\bar{G})+1}, k_{3,1}\right]$,

$k_{3, \Delta(\bar{G})+1} \notin \mathbb{Q}\left[k_{1,1}, k_{1,2}, \ldots, k_{1, \Delta(\bar{G})+1}, k_{2,1}, \ldots, k_{2, \Delta(\bar{G})+1}, k_{3,3}, \ldots, k_{3, \Delta(\bar{G})}\right]$. Since $k_{2, j}-\frac{k_{2,1} k_{1, j}}{k_{1,1}} \neq 0, j=3, \ldots, \Delta(\bar{G})+1$, we can choose these parameters such that $k_{3,1}$ and $k_{3,2}$ are also nonzero. Therefore we get $\left\langle\vec{v}_{1}, \vec{v}_{3}\right\rangle=$ $\left\langle\vec{v}_{2}, \vec{v}_{3}\right\rangle=0$. As a result $\overrightarrow{v_{1}}, \overrightarrow{v_{2}}, \overrightarrow{v_{3}}$ is an orthogonal representation of the induced graph $H=3 K_{1}$ satisfying conditions (i) and (ii).

Rev.Mate.Teor.Aplic. (ISSN print: 1409-2433; online: 2215-3373) Vol. 25(1): 1-28, Jan-Jun 2018 
Case 2. Suppose the induced graph $H$ is $K_{2} \sqcup K_{1}$. Let us assume $v_{1} \nsim v_{2}$, $v_{2} \nsim v_{3}$, and $v_{1} \sim v_{3}$.

The vectors $\vec{v}_{1}$ and $\vec{v}_{2}$ for the vertices $v_{1}$ and $v_{2}$ respectively can be chosen in the same way as in case 1 to get $\left\langle\vec{v}_{1}, \vec{v}_{2}\right\rangle=0$. In order to find a vector $\overrightarrow{v_{3}}=k_{3,1} \overrightarrow{e_{1}}+k_{3,2}{\overrightarrow{e_{2}}}+\cdots+k_{3, \Delta(\bar{G})+1} \vec{e}_{\Delta(\bar{G})+1} \in \mathbb{R}^{\Delta(\bar{G})+1}$ with nonzero components for the vertex $v_{3}$ we know that the vector $\vec{v}_{3}$ should satisfy the system

$$
\begin{aligned}
& \left\langle\overrightarrow{v_{1}}, \overrightarrow{v_{3}}\right\rangle=g_{1}, \quad g_{1} \neq 0 \\
& \left\langle\overrightarrow{v_{2}}, \overrightarrow{v_{3}}\right\rangle=0
\end{aligned}
$$

in the variables $k_{3, j}, j=1,2, \ldots, \Delta(\bar{G})+1$ because $v_{1} \sim v_{3}$ and $v_{2} \nsim v_{3}$ in $G$. Therefore, rewriting the system in the form

$$
\begin{aligned}
& k_{1,1} k_{3,1}+k_{1,2} k_{3,2}+\cdots+k_{1, \Delta(\bar{G})+1} k_{3, \Delta(\bar{G})+1}=g_{1}, \\
& k_{2,1} k_{3,1}+k_{2,2} k_{3,2}+\cdots+k_{2, \Delta(\bar{G})+1} k_{3, \Delta(\bar{G})+1}=0
\end{aligned}
$$

the augmented matrix of the non-homogeneous system becomes

$$
\left(\begin{array}{ccccc|c}
k_{1,1} & k_{1,2} & k_{1,3} & \ldots & k_{1, \Delta}(\bar{G})+1 & g_{1} \\
k_{2,1} & k_{2,2} & k_{2,3} & \ldots & k_{2, \Delta(\bar{G})+1} & 0
\end{array}\right) .
$$

Since $\Delta(\bar{G})+1 \geq 3$, the system has infinitely many solutions depending on one or more free parameters if the system is consistent. Since $k_{1,1}$ is nonzero, we can divide the first row by $k_{1,1}$ to obtain

$$
\begin{gathered}
\left(\begin{array}{ccccc|c}
1 & \frac{k_{1,2}}{k_{1,1}} & \frac{k_{1,3}}{k_{1,1}} & \ldots & \frac{k_{1, \Delta(\bar{G})+1}}{k_{1,1}} & \frac{g_{1}}{k_{1,1}} \\
k_{2,1} & k_{2,2} & k_{2,3} & \ldots & k_{2, \Delta(\bar{G})+1} & 0
\end{array}\right) \sim \\
\left(\begin{array}{cccccc}
1 & \frac{k_{1,2}}{k_{1,1}} & \frac{k_{1,3}}{k_{1,1}} & \ldots & \frac{k_{1, \Delta(\bar{G})+1}}{k_{1} \dot{k}_{1, \Delta(\bar{G})+1} k_{2,1}} & \frac{g_{1}}{k_{1,1}} \\
0 & k_{2,2}-\frac{k_{1,2} k_{2,1}}{k_{1,1}} & k_{2,3}-\frac{k_{1,3} k_{2,1}}{k_{1,1}} & \ldots & k_{2, \Delta(\bar{G})+1}-\frac{g_{1,1}}{k_{1,1}}
\end{array}\right)
\end{gathered}
$$

and since $k_{2,2} \notin \mathbb{Q}\left[k_{1,2}, k_{2,1}, k_{1,1}\right], k_{2,2}-\frac{k_{1,2} k_{2,1}}{k_{1,1}} \neq 0$. Hence we obtain the echelon form of the matrix dividing by $\alpha=k_{2,2}-\frac{k_{1,2} k_{2,1}}{k_{1,1}}$

$$
\left(\begin{array}{cccccc}
1 & \frac{k_{1,2}}{k_{1,1}} & \frac{k_{1,3}}{k_{1,1}} & \ldots & \frac{k_{1, \Delta(\bar{G})+1}}{k_{1,1}} & \frac{g_{1}}{k_{1,1}} \\
0 & 1 & \frac{1}{\alpha}\left(k_{2,3}-\frac{k_{1,3} k_{2,1}}{k_{1,1}}\right) & \ldots & \frac{1}{\alpha}\left(k_{2, \Delta}(\bar{G})+1-\frac{k_{1, \Delta(\bar{G})+1} k_{2,1}}{k_{1,1}}\right) & -\frac{g_{1} k_{2,1}}{\alpha k_{1,1}}
\end{array}\right) .
$$


Choose $k_{3,3} \neq 0, k_{3,4} \neq 0, \ldots, k_{3, \Delta(\bar{G})+1} \neq 0$ one by one from different field extensions $\mathbb{Q}\left[\gamma_{3}\right], \ldots, \mathbb{Q}\left[\gamma_{\Delta(\bar{G})+1}\right]$ such that $\mathbb{Q}\left[\gamma_{i}\right], i=3, \ldots, \Delta(\bar{G})+1$ is not a field extension in the lattice

$$
L\left(k_{1,1}, \ldots, k_{1, \Delta(\bar{G})+1}, k_{2,1}, \ldots, k_{2, \Delta(\bar{G})+1}, \gamma_{3}, \gamma_{4}, \ldots, \gamma_{i-1}\right) .
$$

Therefore, since $g_{1}$ must not be zero, we can choose $g_{1} \in \mathbb{R}$ in such a way that $g_{1}$ does not belong to any of the prior field extensions used so far and satisfy

$$
\begin{aligned}
& \frac{-g_{1} k_{2,1}}{\alpha k_{1,1}}
\end{aligned}
$$

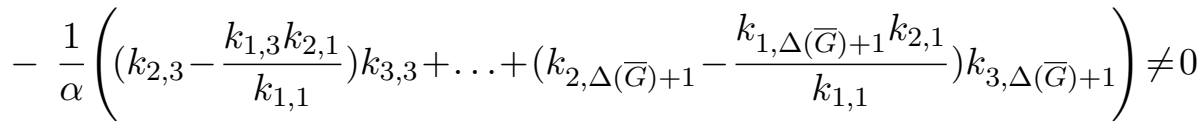

which implies

$$
\begin{aligned}
g_{1} \neq & \frac{-k_{1,1}}{k_{2,1}}\left(\left(k_{2,3}-\frac{k_{1,3} k_{2,1}}{k_{1,1}}\right) k_{3,2}+\cdots+\left(k_{2, \Delta}(\bar{G})+1\right.\right. \\
& \left.\left.-\frac{k_{1, \Delta(\bar{G})+1} k_{2,1}}{k_{1,1}}\right) k_{3, \Delta}(\bar{G})+1\right) .
\end{aligned}
$$

Also

$$
\frac{g_{1}}{k_{1,1}}-\left(\frac{k_{1,2}}{k_{1,1}} k_{3,2}+\frac{k_{1,3}}{k_{1,1}} k_{3,3}+\cdots+\frac{k_{1, \Delta(\bar{G})+1}}{k_{1,1}} k_{3, \Delta(\bar{G})+1}\right) \neq 0
$$

which implies

$$
g_{1} \neq k_{1,1}\left(\frac{k_{1,2}}{k_{1,1}} k_{3,2}+\frac{k_{1,3}}{k_{1,1}} k_{3,3}+\cdots+\frac{k_{1, \Delta(\bar{G})+1}}{k_{1,1}} k_{3, \Delta(\bar{G})+1}\right) .
$$

Thus, choosing $g_{1} \neq 0$ satisfying (3) and (4) we get that the system 1 and 2 is consistent and at least one of its solutions satisfies the adjacency condition and the orthogonal condition for $\vec{v}_{3}$. Also, none of the coordinates of the vectors $\overrightarrow{v_{1}}, \overrightarrow{v_{2}}, \overrightarrow{v_{3}} \in \mathbb{R}^{\Delta(\bar{G})+1}$ are zero. Therefore $\left\{\vec{v}_{1}, \vec{v}_{2}, \vec{v}_{3}\right\}$ is an orthogonal representation in $\mathbb{R}^{\Delta(\bar{G})+1}$ for the induced graph $H=K_{2} \sqcup K_{1}$ satisfying condition (i). Note that if $\vec{v}_{3}=a \vec{v}_{1}, a \in \mathbb{R}$ then $k_{3,1}=a k_{1,1}$ and $k_{3, \Delta(\bar{G})+1}=$ $a k_{1, \Delta(\bar{G})+1}$ which implies that $a=\frac{k_{3,1}}{k_{1,1}}$ and $a=\frac{k_{3, \Delta(\bar{G})+1}}{k_{1, \Delta}(\bar{G})+1}$. As a consequence $k_{3, \Delta}(\bar{G})+1=\frac{k_{3,1}}{k_{1,1}} k_{1, \Delta}(\bar{G})+1$. Hence $k_{3, \Delta(\bar{G})+1} \in \mathbb{Q}\left[k_{1,1}, k_{3,1}, k_{1, \Delta(\bar{G})+1}\right]$ which is a contradiction because $k_{3, \Delta}(\bar{G})+1$ was chosen from a different field extension. Hence, $\vec{v}_{3}$ and $\vec{v}_{1}$ are linearly independent and the vectors $\vec{v}_{1}, \vec{v}_{2}, \vec{v}_{3}$ are pairwise linearly independent satisfying condition (ii). 
Theorem 3.5. Let $G(V, E)$ be a $\delta$-graph then

$$
\operatorname{msr}(G) \leq \Delta(\bar{G})+1=|G|-\delta(G) .
$$

Proof. Let $G(V, E)$ be a $\delta$ graph. Let $Y_{3}\left(\left\{v_{1}, v_{2}, v_{3}\right\}, E_{Y_{3}}\right)$ be the graph induced by the vertices $v_{1}, v_{2}$, and $v_{3}$ which is either $3 K_{1}$ or $K_{2} \sqcup K_{1}$. By Lemma 3.4, $Y_{3}$ has an orthogonal representation in $\mathbb{R}^{\Delta(\bar{G})+1}$.

Also, from Lemma 3.4 we have

(1) The components of vectors in the orthogonal representation of $Y_{3}$ are all nonzero.

(2) $\vec{v} \notin \operatorname{Span}(\vec{u})$ for each pair of distinct vertices $u, v$ in $V_{Y_{3}}$.

From the definition of $\delta$-graph we have

(3) $G(V, E)$ can be constructed starting with $Y_{3}\left(V_{Y_{3}}, E_{Y_{3}}\right)$ and adding one vertex at a time such that the newly added vertex $v_{m}, m \geq 4$ is adjacent to all prior vertices $v_{1}, v_{2}, \ldots, v_{m-1}$ except for at most $\left\lfloor\frac{m}{2}-1\right\rfloor$ vertices.

Applying condition (3) we get a sequence of subgraphs $Y_{3}, Y_{4}, \ldots, Y_{m}, \ldots, Y_{|G|}$ in $G$ induced by $\left\{v_{1}, v_{2}, v_{3}\right\},\left\{v_{1}, v_{2}, v_{3}, v_{4}\right\}, \ldots,\left\{v_{1}, v_{2}, v_{3}, v_{4}, \ldots, v_{m}\right\}, \ldots$, $\left\{v_{1}, v_{2}, v_{3}, v_{4}, \ldots, v_{|G|}\right\}$ respectively.

We will prove that $Y_{j}=\left(V_{j}, E_{j}\right)$ has an orthogonal representation in $\mathbb{R}^{\Delta(\bar{G})+1}$ for all $j=3,4, \ldots,|G|$, satisfying conditions (1) and (2) above. For that purpose, consider the orthogonal representation of $Y_{3}$ satisfying conditions (i) and (ii) given by Lemma 3.4 .

$$
\begin{aligned}
& \overrightarrow{v_{1}}=k_{1,1} \overrightarrow{e_{1}}+k_{1,2} \overrightarrow{e_{2}}+\cdots+k_{1, \Delta(\bar{G})+1} \vec{e}_{\Delta(\bar{G})+1}, \\
& \overrightarrow{v_{2}}=k_{2,1} \overrightarrow{e_{1}}+k_{2,2} \overrightarrow{e_{2}}+\cdots+k_{2, \Delta(\bar{G})+1} \vec{e}_{\Delta(\bar{G})+1}, \\
& \overrightarrow{v_{3}}=k_{3,1} \overrightarrow{e_{1}}+k_{3,2} \overrightarrow{e_{2}}+\cdots+k_{3, \Delta(\bar{G})+1} \vec{e}_{\Delta(\bar{G})+1},
\end{aligned}
$$

where all $k_{i, j}, j=1,2, \ldots, \Delta(\bar{G})+1, i=1,2,3$, are nonzero and are chosen from different field extensions as in the proof of Lemma 3.4. Let $v_{4}$ be a vertex of $G$ such that $v_{4}$ is adjacent to all of $v_{1}, v_{2}, v_{3}$ except at most $\left\lfloor\frac{4}{2}-1\right\rfloor=1$ vertex. Since $G$ and $\bar{G}$ are simple and connected $2 \leq \Delta(\bar{G})$ and therefore $\Delta(\bar{G})+1 \geq 3$.

Since a $\delta$-graph has at least four vertices we make induction over the orthogonal representation of the induced subgraphs obtained from the orthogonal representation of the induced subgraph of three vertices $v_{1}, v_{2}, v_{2}$ of $G$ by adding one vertex at a time. In consecuence, we first prove that a the induced subgraph $Y_{4}$ induced by $\left\{v_{1}, v_{2}, v_{3}, v_{4}\right\}$ has an orthogonal representation in $\mathbb{R}^{\Delta(\bar{G})+1}$. To do this we prove the following claim. 
Claim 3.6. $Y_{4}$ induced by $\left\{v_{1}, v_{2}, v_{3}, v_{4}\right\}$ has an orthogonal representation in $\mathbb{R}^{\Delta(\bar{G})+1}$ satisfying conditions (1) and (2) above.

Proof of claim. We know that

$$
d_{\bar{G}}\left(v_{4}\right) \leq \Delta(\bar{G})<\Delta(\bar{G})+1 .
$$

Since $V_{Y_{3}}=\left\{v_{1}, v_{2}, v_{3}\right\}$ from Lemma 3.4 we have an orthogonal representation for the induced subgraph of $V_{Y_{3}}$ of $G$ in $\mathbb{R}^{\Delta(\bar{G})+1}$ satisfying conditions (1) and (2). We need to find a vector $\overrightarrow{v_{4}}$ for the vertex $v_{4}$ where

$$
\overrightarrow{v_{4}}=k_{4,1} \overrightarrow{e_{1}}+k_{4,2} \overrightarrow{e_{2}}+\cdots+k_{4, \Delta(\bar{G})+1} \vec{e}_{\Delta(\bar{G})+1},
$$

satisfying conditions (1) and (2). Since $v_{4}$ is adjacent with all prior vertices except for at most one of them we have four cases:

1. $v_{4}$ is only adjacent to $v_{1}, v_{2}$ and $v_{3}$ in $G$.

2. $v_{4}$ is only adjacent to $v_{1}$ and $v_{2}$ in $G$.

3. $v_{4}$ is only adjacent to $v_{1}$ and $v_{3}$ in $G$.

4. $v_{4}$ is only adjacent to $v_{2}$ and $v_{3}$ in $G$.

Case 1. $v_{4} \sim v_{1}, v_{4} \sim v_{2}, v_{4} \sim v_{3}$ in $G$.

Choose $k_{4, j}, j=1, \ldots, \Delta(\bar{G})+1$ as follows:

$k_{4,1}=\gamma_{4,1}$ does not belong to any of the field extensions in the lattice of fields $L\left[\mathbb{Q}\left[k_{i, j}\right]\right]$,

$i=1,2,3, j=1,2, \ldots, \Delta(\bar{G})+1$.

$k_{4,2}=\gamma_{4,2}$ does not belong to any of the field extensions in the lattice of field extensions $L\left[\mathbb{Q}\left[k_{i, j}, \gamma_{4,1}\right], i=1,2,3, j=1,2, \ldots, \Delta(\bar{G})+1\right.$.

$k_{4,3}=\gamma_{4,3}$ does not belong to any of the field extensions in the lattice of field extensions $L\left[\mathbb{Q}\left[k_{i, j}, \gamma_{4,1}, \gamma_{4,2}\right]\right], i=1,2,3, j=1,2, \ldots, \Delta(\bar{G})+1$.

Continuing the process until $k_{4, \Delta(\bar{G})+1}$ to obtain

$k_{4, \Delta(\bar{G})+1}=\gamma_{4, \Delta(\bar{G})+1}$ does not belong to any of the field extensions in the lattice of field extensions $L\left[\mathbb{Q}\left[k_{i, j}, \gamma_{4,1}, \gamma_{4,2}, \ldots, \gamma_{4, \Delta(\bar{G})}\right]\right], i=1,2,3$, $j=1,2, \ldots, \Delta(\bar{G})+1$. 
Then $k_{4, j} \neq 0$ for all $j=1,2, \ldots, \Delta(\bar{G})+1$ and $\overrightarrow{v_{4}} \notin \operatorname{Span}\left(\overrightarrow{v_{i}}\right), i=1,2,3$. As a consequence $\left\{\overrightarrow{v_{1}}, \overrightarrow{v_{2}}, \overrightarrow{v_{3}}, \overrightarrow{v_{4}}\right\}$ is an orthogonal representation of $Y_{4}$ at $\mathbb{R}^{\Delta(\bar{G})+1}$. Note that if $\left\langle\vec{v}_{4}, \vec{v}_{1}\right\rangle=0$ then we can solve this equation for $k_{4, \Delta(\bar{G})+1}$ which implies that $k_{4, \Delta(\bar{G})+1} \in L\left[\mathbb{Q}\left[k_{i, j}, \gamma_{4,1}, \gamma_{4,2}, \ldots, \gamma_{4, \Delta(\bar{G})}\right]\right], i=1,2,3$, $j=1,2, \ldots, \Delta(\bar{G})+1$ which is a contradiction. Therefore, $\left\langle\vec{v}_{4}, \vec{v}_{1}\right\rangle \neq 0$. In the same way we can prove that $\left\langle\vec{v}_{4}, \vec{v}_{2}\right\rangle \neq 0$ and $\left\langle\vec{v}_{4}, \vec{v}_{3}\right\rangle \neq 0$.

Case 2. $v_{4} \sim v_{1}, v_{4} \sim v_{2}, v_{4} \nsim v_{3}$ in $G$.

Since $v_{4} \sim v_{1}$ and $v_{4} \sim v_{2}$ and $v_{4} \nsim v_{3}$ then

$$
\begin{aligned}
& \left\langle\overrightarrow{v_{4}}, \overrightarrow{v_{1}}\right\rangle=g_{4,1}, g_{4,1} \neq 0 \\
& \left\langle\overrightarrow{v_{4}}, \overrightarrow{v_{2}}\right\rangle=g_{4,2}, g_{4,2} \neq 0, \\
& \left\langle\overrightarrow{v_{4}}, \overrightarrow{v_{3}}\right\rangle=0 .
\end{aligned}
$$

From these conditions the system $S$ in the variables $k_{4, j}, j=1,2, \ldots, \Delta(\bar{G})+1$ becomes,

$$
\begin{aligned}
& k_{1,1} k_{4,1}+k_{1,2} k_{4,2}+\cdots+k_{1, \Delta(\bar{G})+1} k_{4, \Delta(\bar{G})+1}=g_{4,1}, g_{4,1} \neq 0 \\
& k_{2,1} k_{4,1}+k_{2,2} k_{4,2}+\cdots+k_{2, \Delta(\bar{G})+1} k_{4, \Delta(\bar{G})+1}=g_{4,2}, g_{4,2} \neq 0, \\
& k_{3,1} k_{4,1}+k_{3,2} k_{4,2}+\cdots+k_{3, \Delta(\bar{G})+1} k_{4, \Delta(\bar{G})+1}=0
\end{aligned}
$$

where $k_{i, j}, i=1,2,3, j=1,2, \ldots, \Delta(\bar{G})+1$ were chosen from different field extensions as in the proof of Lemma 3.4. Since

$$
d_{\bar{G}}\left(v_{4}\right) \leq \Delta(\bar{G})<\Delta(\bar{G})+1,
$$

in $\bar{G}$, the number of equations from the orthogonal conditions in the systems are at most $\Delta(\bar{G})<\Delta(\bar{G})+1$, which means that if the non-homogeneous system is consistent then the system will have infinitely many solutions because the system will have at least one free variable. The augmented matrix of the system becomes

$$
\left(\begin{array}{cccc|c}
k_{1,1} & k_{1,2} & \ldots & k_{1, \Delta}(\bar{G})+1 & g_{4,1} \\
k_{2,1} & k_{2,2} & \ldots & k_{2, \Delta}(\bar{G})+1 & g_{4,2} \\
k_{3,1} & k_{3,2} & \ldots & k_{3, \Delta}(\bar{G})+1 & 0
\end{array}\right)
$$

where $g_{4,1} \neq 0, g_{4,2} \neq 0$ are nonzero real numbers.

In order to guarantee that all adjacency conditions are satisfied consider the matrix $3 \times(\Delta(\bar{G})+3)$ below in the variables $k_{4,1}, k_{4,2}, \ldots, k_{4, \Delta(\bar{G})+1},-g_{4,1}$, $-g_{4,2}$. It is possible to consider $-g_{4,1}$ and $-g_{4,2}$ as variables because we only need them to be nonzero. So we can consider them as two additional variables of the homogeneous system $S_{H}$ which has the following augmented matrix: 


$$
\left(\begin{array}{llllll}
k_{1,1} & k_{1,2} & \ldots & k_{1, \Delta(\bar{G})+1} & 1 & 0 \\
k_{2,1} & k_{2,2} & \ldots & k_{2, \Delta(\bar{G})+1} & 0 & 1 \\
k_{3,1} & k_{3,2} & \ldots & k_{3, \Delta}(\bar{G})+1 & 0 & 0
\end{array}\right)
$$

Multiplying the first row by $\frac{1}{k_{1,1}}, k_{1,1} \neq 0$ we get

$$
\left(\begin{array}{cclccc}
1 & \frac{k_{1,2}}{k_{1,1}} & \ldots & \frac{k_{1, \Delta(\bar{G})+1}}{k_{1,1}} & \frac{1}{k_{1,1}} & 0 \\
k_{2,1} & k_{2,2} & \ldots & k_{2, \Delta(\bar{G})+1} & 0 & 1 \\
k_{3,1} & k_{3,2} & \ldots & k_{3, \Delta}(\bar{G})+1 & 0 & 0
\end{array}\right) .
$$

Multiplying the first row by $-k_{2,1}$ and adding the result to the second row and multiplying the first row by $-k_{3,1}$ and adding to the third row we get

$$
\left(\begin{array}{cccccc}
1 & \frac{k_{1,2}}{k_{1,1}} & \ldots & \frac{k_{1, \Delta(\bar{G})+1}}{k_{1,1}} & \frac{1}{k_{1,1}} & 0 \\
0 & k_{2,2}-\frac{k_{1,2} k_{2,1}}{k_{1,1}} & \ldots & k_{2, \Delta}(\bar{G})+1-\frac{k_{1, \Delta}(\bar{G})+1}{k_{1,1}} & -\frac{k_{2,1}}{k_{1,1}} & 1 \\
0 & k_{3,2}-\frac{k_{1,2} k_{3,1}}{k_{1,1}} & \ldots & k_{3, \Delta(\bar{G})+1}-\frac{k_{1, \Delta(\bar{G})+1} k_{3,1}}{k_{1,1}} & 0 & 0
\end{array}\right) .
$$

Let $\alpha=k_{2,2}-\frac{k_{1,2} k_{2,1}}{k_{1,1}}$. Since $k_{2,2} \notin \mathbb{Q}\left[k_{1,1}, k_{1,2}, k_{2,1}\right]$ by construction, $\alpha \neq 0$ and we can continue reducing the matrix to echelon form. Then multiplying the second row by $\frac{1}{\alpha}$ we obtain

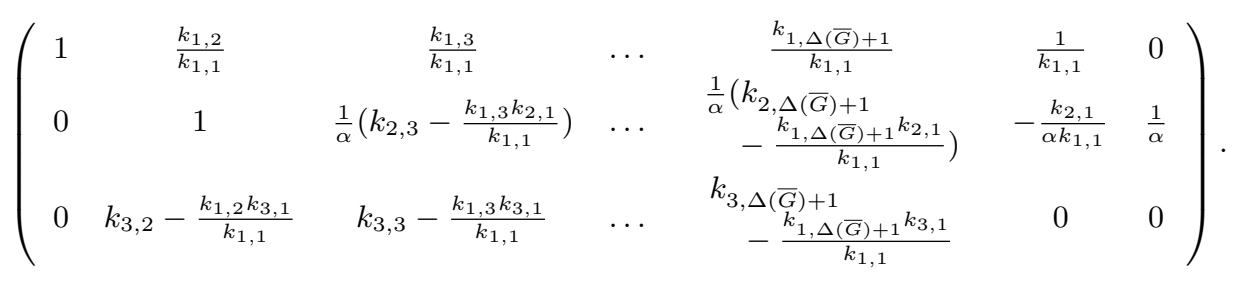

Let $\beta=k_{3,2}-\frac{k_{1,2} k_{3,1}}{k_{1,1}}$. Multiplying the second row by $-\beta$ and adding the result to the third row we get

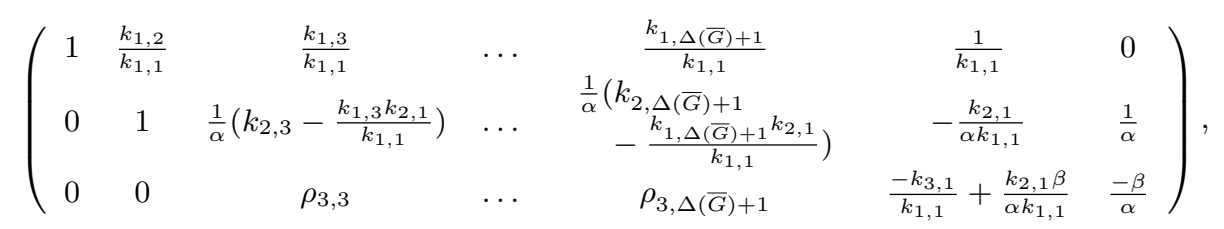

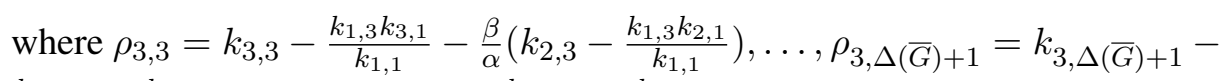
$\frac{k_{1, \Delta}(\bar{G})+1}{k_{3,1}}-\frac{\beta}{\alpha}\left(k_{2, \Delta}(\bar{G})+1-\frac{k_{1, \Delta}(\bar{G})+1}{k_{1,1} k_{2,1}}\right)$. Note that $\rho_{3,3} \neq 0$, otherwise $k_{3,3}$ belongs to a field extension of the lattice $L\left[\mathbb{Q}\left[k_{1,1}, \ldots, k_{1, \Delta}(\bar{G})+1, k_{2,1}, \ldots\right.\right.$, $\left.\left.k_{2, \Delta}(\bar{G})+1, k_{3,1}, k_{3,2}\right]\right]$ which is a contradiction with the definition of $k_{3,3}$. 
Thus, multiplying the third row by $\frac{1}{\rho_{3,3}}$ we obtain the echelon form of the homogeneous system

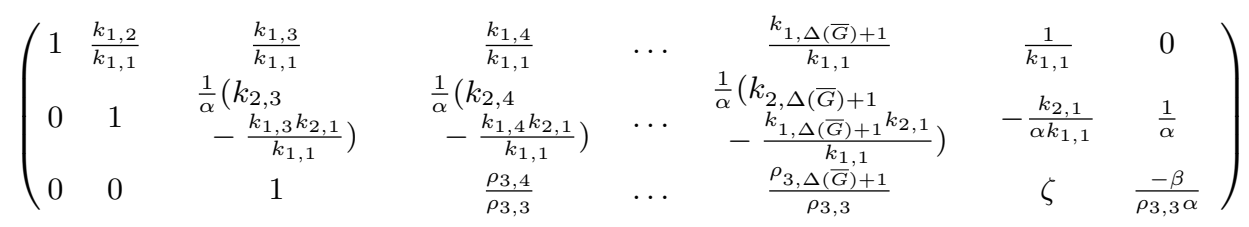

where all the values $\rho_{3, j} \neq 0, j=4, \ldots, \Delta(\bar{G})+1$ as well as $\zeta=\frac{-k_{3,1}}{\rho_{3,3} k_{1,1}}+$ $\frac{k_{2,1} \beta}{\rho_{3,3} \alpha k_{1,1}}$ and $\frac{-\beta}{\rho_{3,3} \alpha}$ are nonzero. Then we can choose values $k_{4,4}, \ldots, k_{4, \Delta(\bar{G})+1}$, $g_{4,1}, g_{4,2}$ nonzero and chosen one by one from different field extensions not in the lattice

$L\left[\mathbb{Q}\left[k_{1,1}\right], \ldots, \mathbb{Q}\left[k_{1, \Delta(\bar{G})+1}\right], \mathbb{Q}\left[k_{2,1}\right], \ldots, \mathbb{Q}\left[k_{2, \Delta(\bar{G})+1}\right], \mathbb{Q}\left[k_{3,1}\right], \ldots, \mathbb{Q}\left[k_{3, \Delta(\bar{G})+1}\right]\right.$

in the following way.

$$
\begin{aligned}
& k_{4,4} \notin \mathbb{Q}\left[k_{i, j}\right], i=1,2,3, j=1, \ldots, \Delta(\bar{G})+1, \\
& k_{4,3} \notin \mathbb{Q}\left[k_{i, j}, k_{4,4}\right], i=1,2,3, j=1, \ldots, \Delta(\bar{G})+1, \\
& \vdots \\
& k_{4, \Delta(\bar{G})+1} \notin \mathbb{Q}\left[k_{i, j}, k_{4,4}, \ldots, k_{4, \Delta(\bar{G})}\right], i=1,2,3, j=1, \ldots, \Delta(\bar{G})+1, \\
& g_{4,1} \notin \mathbb{Q}\left[k_{i, j}, k_{4,4}, \ldots, k_{4, \Delta(\bar{G})+1}\right], i=1,2,3, j=1, \ldots, \Delta(\bar{G})+1, \\
& g_{4,2} \notin \mathbb{Q}\left[k_{i, j}, k_{4,4}, \ldots, k_{4, \Delta(\bar{G})+1}, g_{4,1}\right], i=1,2,3, j=1, \ldots, \Delta(\bar{G})+1,
\end{aligned}
$$

Therefore $k_{4,3}, k_{4,2}$, and $k_{4,1}$ become

$$
\begin{aligned}
& k_{4,3}=-\frac{\rho_{3,4} k_{4,4}}{\rho_{3,3}}+\cdots-\frac{\rho_{3, \Delta(\bar{G})+1} k_{4, \Delta}(\bar{G})+1}{\rho_{3,3}}+\frac{k_{3,1} g_{4,1}}{\rho_{3,3} k_{1,1}}-\frac{k_{2,1} \beta g_{4,1}}{\rho_{3,3} \alpha k_{1,1}}+ \\
& \frac{\beta g_{4,2}}{\rho_{3,3} \alpha} \text {. }
\end{aligned}
$$

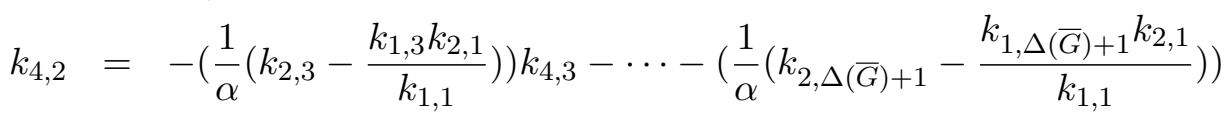

$$
\begin{aligned}
& k_{4, \Delta(\bar{G})+1}+\frac{k_{2,1} g_{4,1}}{\alpha k_{1,1}}-\frac{g_{4,2}}{\alpha} \\
& k_{4,1}=-\frac{k_{1,2} k_{4,2}}{k_{1,1}}-\cdots-\frac{k_{1, \Delta(\bar{G})+1} k_{4, \Delta}(\bar{G})+1}{k_{1,1}}-\frac{g_{4,1}}{k_{1,1}}
\end{aligned}
$$

Note that $k_{4,1}$ depends on $k_{4,2}$ and $k_{4,3}$. Similarly $k_{4,2}$ depends on $k_{4,3}$. Therefore we should choose $k_{4,3}$ first and then back substitute. But $k_{4,3}$ depends on 
$g_{4,1}$ and $g_{4,2}$ which are free variables with the restriction that they cannot be zero. Thus, we can choose $g_{4,1}$ in a field extension not in the lattice of

$Q\left[k_{1,1}, \ldots, k_{1, \Delta(\bar{G})+1}, k_{2,1}, \ldots, k_{2, \Delta(\bar{G})+1}, k_{3,1}, \ldots, k_{3, \Delta(\bar{G})+1}, k_{4,4}, \ldots, k_{4, \Delta(\bar{G})+1}\right]$

and $g_{4,2}$ in some field extension not in the lattice of

$$
Q\left[k_{1,1}, \ldots, k_{1, \Delta}(\bar{G})+1, k_{2,1}, \ldots, k_{2, \Delta}(\bar{G})+1, k_{3,1}, \ldots, k_{3, \Delta}(\bar{G})+1, k_{4,4}, \ldots, k_{4, \Delta}(\bar{G})+1, g_{4,1}\right]
$$

in such a way that $k_{4,3}, k_{4,2}$ and $k_{4,1}$ are nonzero.

As a consequence, the system $S$ is consistent and there exist a solution of values $k_{4, j} \neq 0, j=1,2, \ldots, \Delta(\bar{G})+1$ chosen from different field extensions such that the vector $\overrightarrow{v_{4}}$ satisfies all adjacency conditions and orthogonal conditions with the vectors $\overrightarrow{v_{1}}, \overrightarrow{v_{2}}, \overrightarrow{v_{3}}$. Then the vector $\overrightarrow{v_{4}}=\sum_{j=1}^{\Delta(\bar{G})+1} k_{4, j} \overrightarrow{e_{j}}$ satisfies conditions (1) and (2) and therefore $\left\{\overrightarrow{v_{1}}, \overrightarrow{v_{2}}, \overrightarrow{v_{3}}, \overrightarrow{v_{4}}\right\}$ is an orthogonal representation of $Y_{4}$ in $\mathbb{R}^{\Delta(\bar{G})+1}$.

Case 3. $v_{4} \sim v_{1}, v_{4} \not v_{2}, v_{4} \sim v_{3}$ in $G$.

From the adjacency conditions $v_{4} \sim v_{1}, v_{4} \sim v_{3}$ and orthogonal condition $v_{4} \not v_{2}$ we get the equations:

$$
\begin{aligned}
& \left\langle\overrightarrow{v_{1}}, \overrightarrow{v_{4}}\right\rangle=g_{4,1}, g_{4,1} \neq 0, \\
& \left\langle\overrightarrow{v_{2}}, \overrightarrow{v_{4}}\right\rangle=0, \\
& \left\langle\overrightarrow{v_{3}}, \overrightarrow{v_{4}}\right\rangle=g_{4,2}, g_{4,2} \neq 0 .
\end{aligned}
$$

Interchanging the second and third equations we get a system $S$ similar to case 2. Since all the scalars $k_{i, j}, i=1,2,3, j=1,2, \ldots, \Delta(\bar{G})+1$ are not zero and were chosen from different field extensions the same reasoning as in case 2 applies and the conclusion holds for case 3 .

Case 4. $v_{4} \nsim v_{1}, v_{4} \sim v_{2}, v_{4} \sim v_{3}$ in $G$.

From the adjacency conditions $v_{4} \sim v_{2}, v_{4} \sim v_{3}$ and orthogonal condition $v_{4} \nsim v_{1}$ we get the equations:

$$
\begin{aligned}
& \left\langle\overrightarrow{v_{1}}, \overrightarrow{v_{4}}\right\rangle=0, \\
& \left\langle\overrightarrow{v_{2}}, \overrightarrow{v_{4}}\right\rangle=g_{4,2}, g_{4,2} \neq 0, \\
& \left\langle\overrightarrow{v_{3}}, \overrightarrow{v_{4}}\right\rangle=g_{4,3}, g_{4,3} \neq 0 .
\end{aligned}
$$

Interchanging the first and the third equations we get a system $S$ similar to case 2. Since all the scalars $k_{i, j}, i=1,2,3, j=1,2, \ldots, \Delta(\bar{G})+1$ are not zero and were chosen from different field extensions the same reasoning as in case 2 applies and the conclusion holds for case 4.

As a consequence, in all of the cases we get an orthogonal representation for $Y_{4}$ in $\mathbb{R}^{\Delta(\bar{G})+1}$ satisfying the conditions (1) and (2). This completes of the proof of the claim 3.6. 
Assume that for any $Y_{m-1}=\left(V_{Y_{m-1}}, E_{Y_{m-1}}\right), V_{Y_{m-1}}=\left\{v_{1}, v_{2}, \ldots, v_{m-1}\right\}$ it is possible to get an orthogonal representation of $Y_{m-1}$ in $\mathbb{R}^{\Delta(\bar{G})+1}$. Let $\vec{v}_{1}, \vec{v}_{2}, \ldots, \vec{v}_{m-1}$ be of the form

$$
\vec{v}_{i}=\sum_{j=1}^{\Delta(\bar{G})+1} k_{i, j} \vec{e}_{j},
$$

satisfying conditions (1) and (2) where $k_{i, j} \neq 0$ for all $i=1,2, \ldots, m$, $j=1,2, \ldots, \Delta(\bar{G})+1$, chosen from different field extensions.

We need to prove that if $v_{m}$ is adjoined to $Y_{m-1}$ to get $Y_{m}$ such that $v_{m}$ is adjacent to all prior vertices except at most $\left\lfloor\frac{m}{2}-1\right\rfloor$ vertices then $Y_{m}$ has an orthogonal representation of vectors $\vec{v}_{1}, \vec{v}_{2}, \ldots, \vec{v}_{m}$ in $\mathbb{R}^{\Delta(G)+1}$ satisfying conditions (1) and (2). Assume that $v_{m}$ has an associated vector $\vec{v}_{m}$ such that

$$
\vec{v}_{m}=k_{m, 1} \overrightarrow{e_{1}}+k_{m, 2} \vec{e}_{2}+\cdots+k_{m, \Delta(\bar{G})+1} \vec{e}_{\Delta(\bar{G})+1}
$$

The vertex $v_{m}$ is adjacent to all prior vertices $v_{1}, v_{2}, \ldots, v_{m-1}$ except at most $t \leq\left\lfloor\frac{m}{2}-1\right\rfloor$ vertices in $G$. Then we see that $\vec{v}_{m}$ satisfies at least $m-1-t$ adjacency conditions and $t$ orthogonal conditions.

Let $\rho$ be a permutation of $(1,2, \ldots, m-1)$. Suppose $v_{\rho(1)}, v_{\rho(2)}, \ldots$, $v_{\rho(m-1-t)}$ are adjacent to $v_{m}$ and $v_{\rho(m-t)}, v_{\rho(m-t+1)}, \ldots, v_{\rho(m-2)}, v_{\rho(m-1)}$ are not adjacent to $v_{m}$. The vectors $\vec{v}_{\rho(1)}, \vec{v}_{\rho(2)}, \ldots, \vec{v}_{\rho(m-1-t)}, \vec{v}_{\rho(m-t)}$, $\vec{v}_{\rho(m-t+1)}, \ldots, \vec{v}_{\rho(m-1)}$ and $\vec{v}_{m}$ satisfy the system $S$ given by:

$$
\begin{aligned}
\left\langle\vec{v}_{\rho(1)}, \vec{v}_{m}\right\rangle & =g_{m, 1}, g_{m, 1} \neq 0, \\
\left\langle\vec{v}_{\rho(2)}, \vec{v}_{m}\right\rangle & =g_{m, 2}, \quad g_{m, 2} \neq 0, \\
\vdots & \vdots \\
\left\langle\vec{v}_{\rho(m-1-t)}, \vec{v}_{m}\right\rangle & =g_{m, m-1-t}, \quad g_{m, m-1-t} \neq 0 \\
\left\langle\vec{v}_{\rho(m-t)}, \vec{v}_{m}\right\rangle & =0 \\
\left\langle\vec{v}_{\rho(m-t+1)}, \vec{v}_{m}\right\rangle & =0 \\
\vdots & \vdots \\
\left\langle\vec{v}_{\rho(m-1)}, \vec{v}_{m}\right\rangle & =0
\end{aligned}
$$

containing $m-1-t$ equations from the adjacency conditions and $t$ equations from the orthogonal conditions.

Since the vector $\vec{v}_{\rho(i)}, i=1,2, \ldots, m-1$ has the form

$$
\vec{v}_{\rho(i)}=k_{\rho(i), 1} \vec{e}_{1}+k_{\rho(i), 2} \vec{e}_{2}+\cdots+k_{\rho(i), \Delta(\bar{G})+1} \vec{e}_{\Delta(\bar{G})+1}
$$


where all $k_{\rho(i), j}, i=1,2, \ldots, m-1, j=1,2, \ldots, \Delta(\bar{G})+1$ are not zero and chosen from different field extensions, the system $S$ has the form:

$$
\begin{aligned}
k_{\rho(1), 1} k_{m, 1}+k_{\rho(1), 2} k_{m, 2}+\cdots+k_{\rho(1), \Delta(\bar{G})+1} k_{m, \Delta(\bar{G})+1} & =g_{m, 1} \\
k_{\rho(2), 1} k_{m, 1}+k_{\rho(2), 2} k_{m, 2}+\cdots+k_{\rho(2), \Delta(\bar{G})+1} k_{m, \Delta(\bar{G})+1} & =g_{m, 2} \\
\vdots & \vdots \\
k_{\rho(m-1-t), 1} k_{m, 1}+k_{\rho(m-1-t), 2} k_{m, 2}+\cdots+k_{\rho(m-1-t), \Delta(\bar{G})+1} k_{m, \Delta(\bar{G})+1} & =g_{m, m-1-t} \\
k_{\rho(m-t), 1} k_{m, 1}+k_{\rho(m-t), 2} k_{m, 2}+\cdots+k_{\rho(m-t), \Delta(\bar{G})+1} k_{m, \Delta(\bar{G})+1} & =0 \\
k_{\rho(m-t+1), 1} k_{m, 1}+k_{\rho(m-t+1), 2} k_{m, 2}+\cdots+k_{\rho(m-t+1), \Delta(\bar{G})+1} k_{m, \Delta(\bar{G})+1} & =0 \\
& \vdots \\
k_{\rho(m-1), 1} k_{m, 1}+k_{\rho(m-1), 2} k_{m, 2}+\cdots+k_{\rho(m-1), \Delta(\bar{G})+1} k_{m, \Delta(\bar{G})+1} & =0
\end{aligned}
$$

where $g_{m, 1} \neq 0, g_{m, 2} \neq 0, \ldots, g_{m, m-1-t} \neq 0$. Since $g_{m, 1}, g_{m, 2}, \ldots, g_{m, m-1-t}$ could be any nonzero real numbers satisfying the adjacency conditions we can consider them as additional $m-1-t$ variables under the restriction that they cannot be zero. Therefore we can consider a homogeneous system $S_{H}$ of $m-1$ equations in $m-t+\Delta(\bar{G})$ variables $k_{1}^{m}, k_{2}^{m}, \ldots, k_{\Delta(\bar{G})+1}^{m},-g_{m, 1},-g_{m, 2}$,

$\ldots,-g_{m, m-1-t}$. Now, by hypothesis $t \leq\left\lfloor\frac{m}{2}-1\right\rfloor$. Since $t \leq d_{\bar{G}}\left(v_{m}\right) \leq$ $\Delta(\bar{G})<\Delta(\bar{G})+1$ the homogeneous system $S_{H}$ contains at least one more variable than the number of equations. Hence the system $S_{H}$ given by

$$
\begin{array}{rc}
k_{\rho(1), 1} k_{m, 1}+k_{\rho(1), 2} k_{m, 2}+\cdots+k_{\rho(1), \Delta(\bar{G})+1} k_{m, \Delta(\bar{G})+1}+\left(-g_{m, 1}\right) & =0 \\
k_{\rho(2), 1} k_{m, 1}+k_{\rho(2), 2} k_{m, 2}+\cdots+k_{\rho(2), \Delta(\bar{G})+1} k_{m, \Delta(\bar{G})+1}+\left(-g_{m, 2}\right) & =0 \\
k_{\rho(m-1-t), 1} k_{m, 1}+k_{\rho(m-1-t), 2} k_{m, 2}+\ldots & \vdots \\
+k_{\rho(m-1-t), \Delta(\bar{G})+1} k_{m, \Delta(\bar{G})+1}+\left(-g_{m, m-1-t}\right) & =0 \\
k_{\rho(m-t+), 1} k_{m, 1}+k_{\rho(m-t), 2} k_{m, 2}+\cdots+k_{\rho(m-t), \Delta(\bar{G})+1} k_{m, \Delta(\bar{G})+1} & =0 \\
k_{\rho(m-t+1), 1} k_{m, 1}+k_{\rho(m-t+1), 2} k_{m, 2}+\cdots+k_{\rho(m-t+1), \Delta(\bar{G})+1} k_{m, \Delta(\bar{G})+1} & =0 \\
\vdots & \vdots \\
k_{\rho(m-1), 1} k_{m, 1}+k_{\rho(m-1), 2} k_{m, 2}+\cdots+k_{\rho(m-1), \Delta(\bar{G})+1} k_{m, \Delta(\bar{G})+1} & =0
\end{array}
$$

has infinitely many solutions. Therefore, it is enough to show that there exist at least one solution for $S_{H}$ satisfying the condition that none of the $k_{m, 1}, k_{m, 2}, \ldots$, $k_{m, \Delta(\bar{G})+1}, g_{m, 1}, \ldots, g_{m, m-1-t}$ are zero.

This implies that the system $S$ has a solution which satisfies all adjacency conditions, all orthogonal conditions, and conditions (1) and (2). For that purpose consider the $(m-1) \times(m-t+\Delta(\bar{G}))$ matrix $A$ of the homogeneous system given on the next page. 
Let $\vec{g}=\left(-g_{m, 1},-g_{m, 2}, \ldots,-g_{m, m-1-t}\right)^{T}$. We consider the two cases where $m-1 \leq \Delta(\bar{G})+1$ and $m-1>\Delta(\bar{G})+1$.

Case 1. $m-1 \leq \Delta(\bar{G})+1$ In this case the number of equations in the non-homogeneous system $S$ is at most the number of unknowns $k_{m, 1}, k_{m, 2}, \ldots$, $k_{m, \Delta(\bar{G})+1}$.

$A=$

$\left(\begin{array}{ccccccccccc}k_{\rho(1), 1} & k_{\rho(1), 2} & k_{\rho(1), 3} & \ldots & k_{\rho(1), \Delta(\bar{G})+1} & \mid & 0 & \ldots & 0 & 0 \\ k_{\rho(2), 1} & k_{\rho(2), 2} & k_{\rho(2), 3} & \ldots & k_{\rho(2), \Delta(\bar{G})+1} & \mid & 0 & 1 & \ldots & 0 & 0 \\ k_{\rho(3), 1} & k_{\rho(3), 2} & k_{\rho(3), 3} & \ldots & k_{\rho(3), \Delta(\bar{G})+1} & \mid & 0 & 0 & 1 & \vdots & \vdots \\ \vdots & \vdots & \vdots & \vdots & \vdots & \vdots & \vdots & \vdots & \vdots & \vdots & \vdots \\ k_{\rho(m-t-2), 1} & k_{\rho(m-t-2), 2} & k_{\rho(m-t-2), 3} & \ldots & k_{\rho(m-t-2), \Delta(\bar{G})+1} & 0 & 0 & 0 & 1 & 0 \\ k_{\rho(m-t-1), 1} & k_{\rho(m-t-1), 2} & k_{\rho(m-t-1), 3} & \ldots & k_{\rho(m-t-1), \Delta(\bar{G})+1} & 0 & 0 & 0 & 0 & 1 \\ k_{\rho(m-t), 1} & k_{\rho(m-t), 2} & k_{\rho(m-t), 3} & \ldots & k_{\rho(m-t), \Delta(\bar{G})+1} & 0 & 0 & 0 & 0 & 0 \\ \vdots & \vdots & \vdots & \vdots & \vdots & \vdots & \vdots & \vdots & \vdots & \vdots & \vdots \\ k_{\rho(m-1), 1} & k_{\rho(m-1), 2} & k_{\rho(m-1), 3} & \ldots & k_{\rho(m-1), \Delta(\bar{G})+1} & 0 & 0 & 0 & 0 & 0\end{array}\right)$

Thus $A$ can be row reduced to one of the following two echelon form written in block form:

I.

$$
\begin{aligned}
& B=\left(B_{1} \mid B_{2}\right)
\end{aligned}
$$

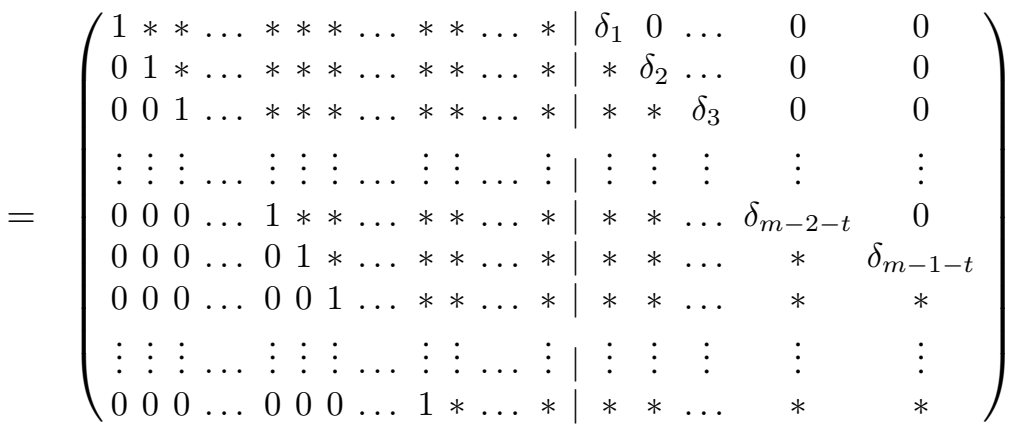

II.

$$
\begin{aligned}
B= & \left(B_{3} \mid B_{2}\right) \\
& =\left(\begin{array}{ccccccc|ccccc}
1 & * & * & \ldots & * & * & \delta_{1} & 0 & \ldots & 0 & 0 \\
0 & 1 & * & \ldots & * & * & * & \delta_{2} & \ldots & 0 & 0 \\
0 & 0 & 1 & \ldots & * & * & * & * & \delta_{3} & 0 & 0 \\
\vdots & \vdots & \vdots & \ldots & \vdots & \vdots & \vdots & \vdots & \vdots & \vdots & \vdots \\
0 & 0 & 0 & \ldots & 1 & * & * & * & \ldots & \delta_{m-t-1} & 0 \\
0 & 0 & 0 & \ldots & 0 & 1 & * & * & \ldots & * & \delta_{m-t}
\end{array}\right) .
\end{aligned}
$$

Rev.Mate.Teor.Aplic. (ISSN print: 1409-2433; online: 2215-3373) Vol. 25(1): 1-28, Jan-Jun 2018 
The matrix $B_{1}$ is a block of size $(m-1) \times(\Delta(\bar{G})+1)$ where $m-1<\Delta(\bar{G})+1$. The matrix $B_{2}$ is a block matrix of size $(m-1) \times(m-1-t)$. Matrix $B_{3}$ is a square matrix of size $m-1(=\Delta(\bar{G})+1)$. In these blocks $*$ denotes a nonzero entry.

Suppose matrix $B$ is of type I. For each vector $\vec{v}_{i}, i=4,5, \ldots, m-1$ the entries are found in field extensions which are not in the lattice of the previous field extensions.

In the block matrix $B_{2}$ we have:

$$
\begin{aligned}
& {\left[B_{2}\right]_{1,1}=\delta_{1}=\frac{1}{k_{\rho(1), 1}} \neq 0,} \\
& {\left[B_{2}\right]_{2,2}=\delta_{2}=\left(k_{\rho(2), 2}-\frac{k_{\rho(1), 2} \cdot k_{\rho(2), 1}}{k_{\rho(1), 1}}\right)^{-1} \neq 0,} \\
& {\left[B_{2}\right]_{3,3}=\delta_{3}=\frac{1}{\alpha} \neq 0, \alpha \in Q\left[k_{\rho(1), 1}, k_{\rho(1), 2}, k_{\rho(1), 3}, k_{\rho(2), 1}, k_{\rho(2), 2}, k_{\rho(2), 3},\right.} \\
& \left.k_{\rho(3), 1}, k_{\rho(3), 2}, k_{\rho(3), 3}\right] .
\end{aligned}
$$

Continuing this process we get that all the entries on the diagonal of $B_{2}$ to be nonzero. So all the rows of $B_{2}$ have at least one entry nonzero. Thus,

$\left[B_{2} \vec{g}\right]_{1}=-\delta_{1} \cdot g_{m, 1}=\frac{-g_{m, 1}}{k_{1,1}}$. Choosing $g_{m, 1}$ not in the lattice generated by the previous field extensions for $k_{i, j}, i=1,2, \ldots, m-1$, $j=1,2, \ldots, \Delta(\bar{G})+1$, we get $\left[B_{2} \vec{g}\right]_{1} \neq 0$.

$\left[B_{2} \vec{g}\right]_{2}=\alpha_{2,1} g_{m, 1}+\delta_{2} g_{m, 2}, \alpha_{2,1} \neq 0, \delta_{2} \neq 0$. Then we can choose a value for $g_{m, 2}$ from a field extension not in the lattice of fields generated by the previous values $k_{i, j}, i=1,2, \ldots, m-1, j=1,2, \ldots, \Delta(G)+1, g_{m, 1}$ so that $\left[B_{2} \vec{g}\right]_{2} \neq 0$.

$\left[B_{2} \vec{g}\right]_{3}=\alpha_{3,1} g_{m, 1}+\alpha_{3,2} g_{m, 2}+\delta_{3} g_{m, 3}, \alpha_{3,1} \neq 0, \delta_{3} \neq 0$. As above we can choose $g_{m, 3} \neq 0$ and such that $\left[B_{2} \vec{g}\right]_{3} \neq 0$ by taking $g_{m, 3}$ neither in $Q\left[\frac{\alpha_{3,1} g_{m, 1}+\alpha_{3,2} g_{m, 2}}{-\delta_{3}}\right]$ nor in any of the previous field extensions.

Continuing this process and applying similar choices we see that $g_{m, 4}, g_{m, 5}, \ldots$, $g_{m, m-1}$ can be chosen nonzero. Moreover, matrix $B_{1}$ shows that there is at least one free variable for the solution of $k_{m, j}, j=1,2, \ldots, \Delta(\bar{G})+1$. All of these free variables can be chosen in different field extensions such that all other unknowns are not zero. Otherwise it is possible to show that the last choice belongs to the field containing all the previous chosen values which is a contradiction. Now, suppose that $k_{m, 1}, k_{m, 2}, \ldots, k_{m, r}, r<\Delta(\bar{G})+1$ can be written in terms of $k_{m, r+1}, \ldots, k_{m, \Delta(\bar{G}+1)}$ as

$$
\begin{aligned}
& k_{m, i}=\alpha_{r+1} k_{m, r+1}+\cdots+\alpha_{\Delta(\bar{G})+1} k_{m, \Delta(\bar{G})+1}+\varphi_{i}\left(g_{m, 1}, g_{m, 2}, \ldots, g_{m, m-1}\right), \\
& i=1,2, \ldots, r
\end{aligned}
$$


where $\alpha_{r+1}, \ldots, \alpha_{\Delta(\bar{G})+1}$ are all nonzero and $\varphi_{i}$ is a linear combination of $g_{m, 1}, \ldots, g_{m, m-1}$. Also $\varphi_{i}\left(g_{m, 1}, g_{m, 2}, \ldots, g_{m, m-1}\right) \neq 0, i=1,2, \ldots, r$. Thus, by choosing values $k_{m, r+1}, \ldots, k_{m, \Delta(\bar{G})+1}$ in different field extensions and substituting them in 6 , we obtain that $k_{m, i} \neq 0, i=1,2, \ldots, r$.

As a consequence, the vector $\vec{v}_{m}$ exists and all of its entries are nonzero.

If matrix $B$ is of type II we apply same process as in case of type I. Again, we can obtain the vector $v_{m}$ having all its entries nonzero and $g_{m, i} \neq 0$ for $i=1, \ldots, m-1$.

Case 2. $m-1>\Delta(\bar{G})+1$

In this case the number of equations in the non-homogeneous system $S$ is more than the number of unknowns $k_{m, 1}, k_{m, 2}, \ldots, k_{m, \Delta}(\bar{G})+1$.

We need to analyze three possible subcases where $m-1-t<\Delta(\bar{G})+$ $1, m-1-t=\Delta(\bar{G})+1$ or $m-1-t>\delta(\bar{G})+1$.

1. If $m-1-t<\Delta(\bar{G})+1$ then matrix $B$ has the form

$$
B=\left(\begin{array}{cccccc|ccc|cccccccc}
1 & * & * & \ldots & * & * & \ldots & * & * & 0 & 0 & 0 & 0 & \ldots & 0 & 0 \\
0 & 1 & * & \ldots & * & * & \ldots & * & * & * & 0 & 0 & 0 & \ldots & 0 & 0 \\
0 & 0 & 1 & \ldots & * & * & \ldots & * & * & * & * & 0 & 0 & \ldots & 0 & 0 \\
\vdots & \vdots & \vdots & \ldots & \vdots & \vdots & \ldots & \vdots & \vdots & \ldots & \vdots & \vdots & \vdots & \vdots & \vdots & \vdots \\
0 & 0 & 0 & \ldots & 1 & * & \ldots & * & * & \ldots & * & * & * & \ldots & * & * \\
- & - & - & - & - & - & - & \ldots & - & - & - & - & - & - & \ldots & - & - \\
0 & 0 & 0 & \ldots & 0 & 1 & \ldots & * & * & \ldots & * & * & * & \ldots & * & * \\
\vdots & \vdots & \vdots & \vdots & \vdots & \vdots & \ldots & \vdots & \vdots & \ldots & \vdots & \vdots & \vdots & \vdots & \vdots & \vdots \\
0 & 0 & 0 & \ldots & 0 & 0 & \ldots & * & * & \ldots & * & * & * & \ldots & * & * \\
0 & 0 & 0 & \ldots & 0 & 0 & \ldots & 1 & * & \ldots & * & * & * & \ldots & * & * \\
0 & 0 & 0 & \ldots & 0 & 0 & \ldots & 0 & * & \ldots & * & * & * & \ldots & * & * \\
\vdots & \vdots & \vdots & \vdots & \vdots & \vdots & \ldots & \vdots & \vdots & \ldots & \vdots & \vdots & \vdots & \vdots & \vdots & \vdots \\
0 & 0 & 0 & \ldots & 0 & 0 & \ldots & 0 & * & \ldots & * & * & * & \ldots & * & *
\end{array}\right)
$$

The matrix $B_{1}$ is a square matrix $(m-1-t) \times(m-1-t)$, matrix $B_{2}$ has size $(m-1-t) \times(\Delta(\bar{G})+2+t-m)$, matrix $B_{3}$ is a square matrix of size $m-1-t$. Matrix $B_{4}$ is a zero matrix of size $t \times m-1-t$. The central blocks $B_{2}, B_{5}$ form a block of size $(m-1) \times(\Delta(\bar{G})+2+t-m)$ and corresponds to the columns of free variables $k_{m, m-t}, \ldots, k_{m, \Delta}(\bar{G})+1$ of the system $S$. The block $B_{6}$ has size $t \times(m-1-t)$. 
Consider the block matrix $\left(B_{5} B_{6}\right)$ of size $t \times(\Delta(\bar{G})+1)$. Recalling that the value $t$ is the number of orthogonal conditions for $v_{m}$ in $G$ which is equivalent to $\mathrm{d}_{\bar{G}}\left(v_{m}\right)$ we get $t \leq \Delta(\bar{G})<\Delta(\bar{G})+1$. As a consequence, the homogeneous system $\left(B_{5} B_{6}\right) \vec{w}=0$ where $\vec{w}$ is a vector of size $(\Delta(\bar{G})+1) \times 1$ in the variables $k_{m, m-t}, \ldots, k_{m, \Delta(\bar{G})+1},\left(-g_{m, 1}\right), \ldots$, $\left(-g_{m, m-1-t}\right)$, has infinitely many solutions depending on at least one free variable. Choosing these free variables in different field extensions as we did previously, we get nonzero values for $k_{m, m-t}, \ldots, k_{m, \Delta(\bar{G})+1}, g_{m, 1}$, $\ldots, g_{m, m-1-t}$. We get the values of the remaining unknowns $k_{m, i}, i=$ $1,2, \ldots, m-1-t$ of the system $S$ applying back substitution.

Since all the entries with $*$ in the block $B_{1}$ are nonzero and belong to different field extensions, the values $k_{m, i}, i=1,2, \ldots, m-1-t$ are also nonzero.

As a consequence, the non-homogeneous system $S$ is consistent and the vector $v_{m}$ with no zero entries exists.

2. If $m-1-t=\Delta(\bar{G})+1$ then the matrix $B$ has the form

$$
\begin{aligned}
& B=\left(\begin{array}{ccc}
B_{1} & \mid & B_{2} \\
- & - \\
0 & \mid & R
\end{array}\right)
\end{aligned}
$$

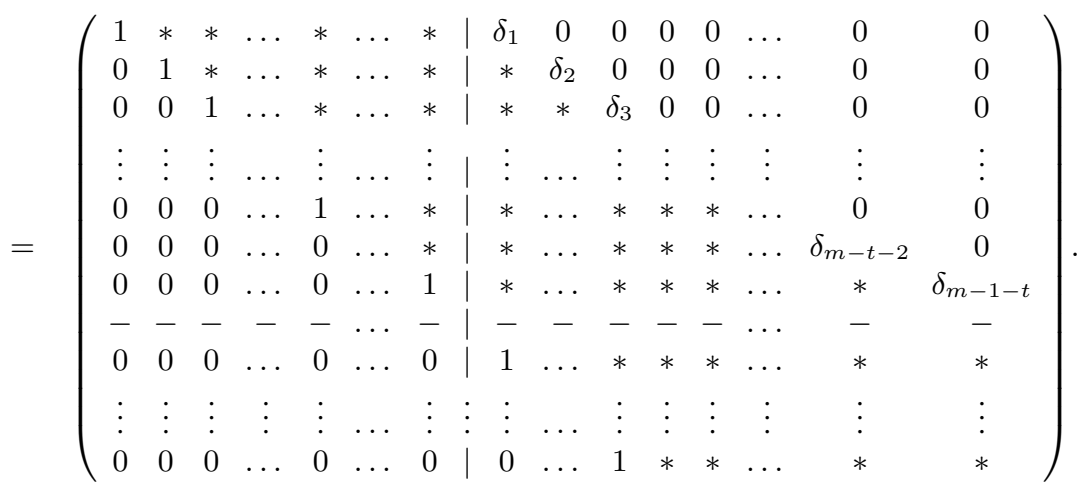

In this case the matrices $B_{1}$ and $B_{2}$ are square matrices of size $m-1-t=(\Delta(\bar{G})+1)$. The matrix $R$ has size $t \times m-1-t$. Since $t<\left\lfloor\frac{m}{2}-1\right\rfloor$ we get that $2 t<m-2<m-1$ which implies that $t<m-1-t$.

Therefore the system $R \vec{g}=0$ has infinitely many solutions with $m-1-2 t$ free variables. Taking the free variables from different field extensions we get all the values $g_{m, 1}, \ldots, g_{m, m-1-t}$ nonzero. Substituting $g_{m, 1}, \ldots, g_{m, m-1-t}$ in the equations of the system $B_{2} \vec{g}=0$ we get 
$\left[B_{2} \vec{g}\right]_{i} \neq 0$ for all $i=1, \ldots, m-1-t$. Otherwise, it is possible to show that the last choice belongs to the field containing all previous chosen values which is a contradiction. This implies that $k_{m, \Delta(\bar{G})+1}=$ $\left[B_{2} \vec{g}\right]_{m-1-t} \neq 0$ from the last row of $\left(B_{1} B_{2}\right)$.

Applying back substitution and similar argument with $g_{m, 1}, \ldots, g_{m, m-1-t}$ we conclude that $k_{m, 1}, \ldots, k_{m, \Delta}(\bar{G})$ are also nonzero. Thus the system $S$ has a solution with nonzero values for the unknowns. As a consequence there exists a vector $\vec{v}_{m}$ satisfying all the adjacency conditions and orthogonal conditions.

3. $m-1-t>\Delta(\bar{G})+1$ then matrix $B$ has the form

$$
\begin{aligned}
B= & \left(\begin{array}{ccc}
B_{1} & \mid & B_{2} \\
- & & - \\
0 & \mid & R
\end{array}\right) \\
= & \left(\begin{array}{cccccccc|cccccccc}
1 & * & * & \ldots & * & \ldots & * & * & 0 & 0 & 0 & 0 & \ldots & 0 & 0 \\
0 & 1 & * & \ldots & * & \ldots & * & * & * & 0 & 0 & 0 & \ldots & 0 & 0 \\
0 & 0 & 1 & \ldots & * & \ldots & * & * & * & * & 0 & 0 & \ldots & 0 & 0 \\
\vdots & \vdots & \vdots & \ldots & \vdots & \ldots & \vdots & \mid & \vdots & \ldots & \vdots & \vdots & \vdots & \vdots & \vdots & \vdots \\
0 & 0 & 0 & \ldots & 1 & \ldots & * & \mid & * & \ldots & * & * & * & \ldots & 0 & 0 \\
\vdots & \vdots & \vdots & \vdots & \vdots & \ldots & \vdots & \vdots & \vdots & \ldots & \vdots & \vdots & \vdots & \vdots & \vdots & \vdots \\
0 & 0 & 0 & \ldots & 0 & \ldots & * & & * & \ldots & * & * & * & \ldots & * & 0 \\
0 & 0 & 0 & \ldots & 0 & \ldots & 1 & & * & \ldots & * & * & * & \ldots & * & * \\
- & - & - & - & - & \ldots & - & - & - & - & - & - & \ldots & - & - \\
0 & 0 & 0 & \ldots & 0 & \ldots & 0 & \mid & 1 & \ldots & * & * & * & \ldots & * & * \\
\vdots & \vdots & \vdots & \vdots & \vdots & \ldots & \vdots & \vdots & \vdots & \ldots & \vdots & \vdots & \vdots & \vdots & \vdots & \vdots \\
0 & 0 & 0 & \ldots & 0 & \ldots & 0 & \mid & 0 & \ldots & 1 & * & * & \ldots & * & *
\end{array}\right) .
\end{aligned}
$$

The matrix $B_{1}$ has size $(m-1-t) \times(\Delta(\bar{G})+1+r), 0<r<t$ where $m-1-t=\Delta(\bar{G})+1+r$. This matrix contains the columns of the unknowns $k_{m, 1}, \ldots, k_{m, \Delta(\bar{G})+1},\left(-g_{m, 1}\right), \ldots,\left(-g_{m, r}\right)$. The matrix $B_{2}$ has size $(m-1-t) \times(\Delta(\bar{G})+1)$. The 0 matrix has size $t \times(\Delta(\bar{G})+$ $1+r)$. The matrix $R$ has size $t \times(\Delta(\bar{G})+1)$.

Since $t \leq \Delta(\bar{G})<\Delta(\bar{G})+1$ the system $R \vec{g}=0$ has infinitely many solutions with at least one free variable. By the same argument as in case 2 we get that the system $S$ is consistent and the solution with nonzero values for $k_{m, 1}, \ldots, k_{m, \Delta}(\bar{G})+1$ gives a vector $\vec{v}_{m}$ which satisfies all the adjacency conditions and orthogonal conditions. 
Hence, $Y_{m}$ has an orthogonal representation of vectors in $\mathbb{R}^{\Delta(\bar{G})+1}$ satisfying the conditions (1) and (2). Thus $Y_{|G|}=G$ has an orthogonal representation in $\mathbb{R}^{\Delta(\bar{G})+1}$ satisfying conditions (1) and (2).

Using the same argument in the construction of vector $v_{4}$, we prove that $v_{1}, \ldots, v_{m}$ is pairwise linearly independent set of vectors in $\mathbb{R}^{\Delta(\bar{G})+1}$. Finally since $\operatorname{msr}(G)$ is the smallest dimension in which $G$ has an orthogonal representation $\operatorname{msr}(G) \leq \Delta(\bar{G})+1$. Since $\delta(G)+\Delta(\bar{G})=|G|-1$ we conclude that $G$ satisfies the delta conjecture, namely, $\operatorname{msr}(G) \leq|G|-\delta(G)$. This completes the proof of the main theorem.

Observation 3.7. In the construction of orthogonal representation of the induced graph $Y_{m}$ it is sufficient to consider $t \leq\left\lfloor\frac{m}{2}-1\right\rfloor$ if $m$ is even and $t<\left\lfloor\frac{m-1}{2}\right\rfloor$ if $m$ is odd. In both cases we obtain the condition $t<(m-1-t)$ that we need to get infinitely many solutions for the system $R \vec{g}=0$. This difference in the upper bounds for $t$ is important for small values of $m$ but for larger values of $m$ these upper bounds are asymptotically equivalent. However, it means that we could get an orthogonal representation of pairwise linearly independent vectors in $\mathbb{R}^{\Delta(\bar{G})+1}$ for some graphs which are not necessarily $\delta$-graphs.

Observation 3.8. Reducing the matrix A to an echelon form needs a finite number of operations as well as reducing the matrix $R$ to an echelon form. It means that all the values $k_{i, j}, i=1,2, \ldots, m-1, j=1,2, \ldots, \Delta(\bar{G})+1$ can be chosen from different field extensions in such a way that all the values $*$ in the reduced echelon form of $A$ are nonzero and belong to different field extensions.

Observation 3.9. The condition of choosing values $k_{i, j}, i=1,2, \ldots,|G|$, $j=1,2, \ldots, \Delta(\bar{G})+1$ from different field extensions was imposed to guarantee the consistency of the non-homogeneous system S. Also, we use this nonzero entries of the vectors $\vec{v}_{1}, \ldots, \vec{v}_{m-1}$ to guarantee the adjacency conditions and orthogonal conditions of the vector $\vec{v}_{m}$ corresponding to the newly added vertex. But calculating the orthogonal representation using this approach could be time consuming. Since we know that it is possible to get an orthogonal representation of $\delta$-graph $G$ in $\mathbb{R}^{\Delta(\bar{G})+1}$ and since the representation is not unique, it may be possible to calculate the orthogonal representation using integers or rational numbers. However, calculating the orthogonal representation of a $\delta$-graph $G$ in this way could also be tedious because we may need to apply a backtracking procedure during the calculation due to some adjacency conditions of the vector corresponding to the newly added vertex may not be satisfied. When that happens, we may need to go back to some of the previous vectors and recalculate them until we fix the adjacency conditions. 


\section{Examples of $\delta$-graphs and their msr}

The result proved above give us a huge family of graph which satisfies delta conjecture. Since, the complement of a C-delta graphs is a $\delta$-graph, it is enough to identify a $\mathrm{C}$ - $\delta$-graph and therefore we know that its complement is a $\delta$-graph satisfying delta conjecture.

Some examples of C- $\delta$ graphs that we can find in [15] are the Cartesian Product $K_{n} \square P_{m}, n \geq 3, m \geq 4$, Mobiüs Lader $M L_{2 n}, n \geq 3$, Supertriangles $T n, n \geq 4$, Coronas $S_{n} \circ P_{m}, n \geq 2, m \geq 1$ where $S_{n}$ is a star and $P_{m}$ a path, Cages like Tutte's $(3,8)$ cage, Headwood's $(3,6)$ cage and many others, Blanusa Snarks of type 1 and 2 with 26,34, and 42 vértices, Generalized Petersen Graphs $G p 1$ to $G p 16$, and many others.

In order to show the technique used in the proved result consider the following example.

Example 4.1. If $G$ is the Robertson's $(4,5)$-cage on 19 vertices then it is a 4regular C- $\delta$ graph. Since $\Delta(G)=4$, the $\operatorname{msr}(G) \leq 5$. To see this is a C- $\delta$ graph it is enough to label its vertices in the way shown in Figure 2.

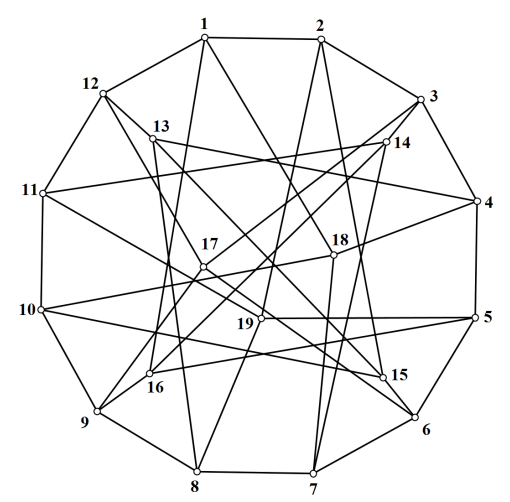

Figure 2: B.2 Robertson's (4,5)-cage (19 vertices).

\section{Conclusion}

The result proved above give us a tool to identify a wide range of families of graphs which satisfy delta conjecture. The techniques used in the proof could be used in future research as a new approach to solve delta conjecture. However, it is clear that the main problem is still open. 


\section{Acknowledgment}

I would like to thanks my adviser Dr. Sivaram Narayan for his guidance and suggestions of this research. Also I want to thank the Mathematics Department of Universidad de Costa Rica and Universidad Nacional Estatal a Distancia because of their sponsorship during my dissertation research. Specially thanks to the Mathematics Department of Central Michigan University where I did the research for this paper.

\section{References}

[1] AIM Minimum Rank-Special Graphs Work Group (Barioli, B.; Barrett, W.; Butler, S.; Cioaba, S.; Fallat, S.; Godsil, C.; Haemers, W.; Hogben, L.; Mikkelson, R.; Narayan, S.; Pryporova, O.; Sciriha, I.; Stevanovic, D.; Van Der Holst, H.; Van Der Meulen, K.; Wangsness, A.) (2008) "Zero forcing sets and the minimum rank of graphs", Linear Algebra and its Applications 428(7): 1628-1648.

[2] Barioli, F.; Barrett, W.; Fallat, S.; Hall, H.; Hogbend, L.; van der Holst, H. (2012) "On the graph complement conjecture for minimum rank", Linear Algebra and its Applications 436(12): 4373-4391.

[3] Barioli, F.; Barrett, W.; Fallat, S.; Hall, H.; Hogbend, L.; Shader, B.; van den Driessche, P.; van der Holst, H. (2010) "Zero forcing parameters and minimum rank problems", Linear Algebra and its Applications 433(2): $401-411$.

[4] Barioli, F.; Fallat, S.; Mitchell, L.; Narayan, S. (2011) "Minimum semidefinite rank of outerplanar graphs and the tree cover number", Electronic Journal of Linear Algebra 22(2): 10-21.

[5] Barrett, W.; van der Holst, H.; Loewy, R. (2004) "Graphs whose minimal rank is two", Electronic Journal of Linear Algebra 11(21): 258-280.

[6] Beagley, J et al . (2007) "On the Minimum Semidefinite Rank of a Graph Using Vertex Sums, Graphs with $\operatorname{msr}(G)=|G|-2$, and the msrs of Certain Graphs Classes", in: NSF-REU Report from Central Michigan University (Summer 2007).

[7] Berman, A.; Friedland, S.; Hogben, L.; Rothblum, U.; Shader, B. (2008) "An upper bound for the minimum rank of a graph", Linear Algebra and its Applications 429(7): 1629-1638. 
[8] Bollobás, B. (1998) Modern Graph Theory. Springer, Memphis, TN.

[9] Bondy, A.; Murty, M. (2008) Graph Theory. Springer, San Francisco, CA.

[10] Booth, M.; Hackney, P.; Harris, B.; Johnson, C.; Lay, M.; Lenker, T.; Mitchell, L.; Narayan, S.; Pascoe, A.; Sutton, B. (2011) "On the minimum semidefinite rank of a simple graph", Linear and Multilinear Algebra 59(5): 483-506.

[11] Booth, M.; Hackney, P.; Harris, B.; Johnson, C.R.; Lay, M.; Mitchell, L.H.; Narayan, S.K.; Pascoe, A.; Steinmetz, K.; Sutton, B.D.; Wang, W. (2008) "On the minimum rank among positive semidefinite matrices with a given graph", SIAM Journal on Matrix Analysis and Applications 30(2): 731740.

[12] Brandstädt, A.; Spinrad, J.; Le, V. (1999) Graph Classes: A Survey. SIAM Monographs on Discrete Mathematics and Applications, Philadelphia, PA.

[13] Brualdi, R.; Leslie, H.; Shader, B. (2007) AIM workshop spectra of families of matrices described by graphs, digraphs, and sign patterns final report: mathematical results, en: http://www.aimath.org/ pastworkshops/matrixspectrumrep.pdf

[14] Chartrand, G.; Lesniak, L.; Zhang, P. (2010) Graphs \& Digraphs. Taylor $\&$ Francis Group, Boca Raton, FL.

[15] Diaz, P. (2014) On the Delta Conjecture and the Graph Complement Conjecture for Minimum Semidefinite Rank of a Graph. Ph.D Dissertation, Mathematics Department, Central Michigan University, Michigan.

[16] Ekstrand, J.; Erickson, C.; Hall, H.; Hay, H.; Hogben, L.; Johnson, R.; Kingsley, N.; Osborne, S.; Peters, T.; Roat, J.; Ross, A.; Row, D.; Warnberg, N.; Young, M. (2013) "Positive semidefinite zero forcing", Linear Algebra and its Applications 439(7): 1862-1874.

[17] Fallat, S.M.; Hogben, L.(2007) "The minimum rank of symmetric matrices described by a graph: a survey", Linear Algebra and its Applications 426(23): 558-582.

[18] Hackney, P.; Harris, B.; Lay, M.; Mitchell, L.H.; Narayan, S.K.; Pascoe, A. (2009) "Linearly independent vertices and minimum semidefinite rank", Linear Algebra and its Applications 431(8): 1105-1115. 
[19] Holst, H. (2003) "Graphs whose positive semidefinite matrices have nullity at most two", Linear Algebra and its Applications 375: 1-11.

[20] Horn, R,; Johnson, C. (1985) Matrix Analysis. Cambridge University Press, England.

[21] Hogben, L. (2010) "Minimum rank problems", Linear Algebra and its Applications 432(8): 1961-1974.

[22] Hogben, L. (2008) "Orthogonal representations, minimum rank, and graph complements", Linear Algebra and its Applications 428(11-12): 25602568 .

[23] Jianga, Y.; Mitchell, L.H.; Narayan, S.K.(2008) "Unitary matrix digraphs and minimum semidefinite rank", Linear Algebra and its Applications 428(7): 1685-1695.

[24] Mitchell L.(2011) "On the graph complement conjecture for minimum semidefinite rank", Linear Algebra and its Applications 435(6): 13111314.

[25] Mitchell, L.H.; Narayan, S.K.; Zimmerc, A.M. (2010) "Lower bounds in minimum rank problems", Linear Algebra and its Applications 432(1): $430-440$.

[26] Narayan, S.; Sharawi, Y. (2014) "Bounds on minimum semidefinite rank of graphs", Linear and Multilinear Algebra 63(4): 774-787.

[27] Nylen, P.M.(1996) "Minimum-rank matrices with prescribed graph", Linear Algebra and its Applications 248(15): 303-316.

[28] Peters, T. (2012) "Positive semidefinite maximum nullity and zero forcing number", Electronic Journal of Linear Algebra 23(): 815-830.

[29] Read,R.; Wilson, R. (1998) An Atlas of Graphs. Oxford University Press, Reino Unido.

[30] Sharawi, Y. (2011) Minimum Semidefinite Rank of a Graph. Ph.D. Dissertation, Mathematics Department, Central Michigan University, Michigan.

[31] West, D.B. (1996) Introduction to Graph Theory. Prentice Hall Inc, Estados Unidos. 